ICRR-Report-513-2004-11

YITP-04-73

October 22, 2018

\title{
Non-Perturbative Effect on Dark Matter Annihilation and Gamma Ray Signature from Galactic Center
}

\author{
Junji Hisano $^{a}$, Shigeki. Matsumoto ${ }^{a}$, Mihoko M. Nojiri ${ }^{b}$, and \\ Osamu Saito ${ }^{a}$ \\ ${ }^{a}$ ICRR, University of Tokyo, Kashiwa 277-8582, Japan \\ ${ }^{b}$ YITP, Kyoto University, Kyoto 606-8502, Japan
}

\begin{abstract}
Detection of gamma rays from dark matter annihilation in the galactic center is one of the feasible techniques to search for dark matter. We evaluate the gamma ray flux in the case that the dark matter has an electroweak $\mathrm{SU}(2)_{L}$ charge. Such dark matter is realized in the minimal supersymmetric standard model (MSSM) when the lightest SUSY particle is the Higgsino- or Wino-like neutralino. When the dark matter is heavy compared to the weak gauge bosons, the leading-order calculation of the annihilation cross sections in perturbation breaks down due to a threshold singularity. We take into account non-perturbative effects by using the non-relativistic effective theory for the two-body states of the dark matter and its $\mathrm{SU}(2)_{L}$ partner(s), and evaluate precise cross sections relevant to the gamma ray fluxes. We find that the annihilation cross sections may be enhanced by several orders of magnitude due to resonances when the dark matter mass is larger than $1 \mathrm{TeV}$. Furthermore, the annihilation cross sections in the MSSM may be changed by factors even when the mass is about $500 \mathrm{GeV}$. We also discuss sensitivities to gamma ray signals from the galactic center in the GLAST satellite detector and the large Air Cerenkov Telescope arrays.
\end{abstract}




\section{Introduction}

Recent cosmological observations determine precisely the mean densities of matter and baryon in the Universe [1, and existence of non-baryonic dark matter is established. Weakly interacting massive particles (WIMPs) are considered to be good candidates of the dark matter [2]. They act as the cold dark matter in the structure formation of the universe. High resolution $N$-body simulations show that the cold dark matter hypothesis explains well the structure larger than about $1 \mathrm{Mpc}$ [3]. On the other hand, fundamental problems, such as (i) the constituent of the dark matter and the origin in the thermal history and (ii) the dark matter distribution in the galactic scale, are not still resolved. It is important to detect the dark matter in direct or indirect methods in order to answer the questions.

Many detection methods have been proposed and some of the experiments are now operating. Among those, the detections of exotic cosmic ray fluxes, such as positrons, anti-protons and gamma rays, are feasible techniques to search for the dark matter particles [4]-[9]. In particular, an excess of monochromatic (line) gamma rays due to the pair annihilation would be a robust signal if observed, because diffused gamma ray background must have a continuous energy spectrum [9]. The GLAST satellite detector [10] and the large Atmospheric Cerenkov Telescope (ACT) arrays, such as CANGAROO III [11], HESS [12, MAGIC [13] and VERITAS [14, can search for the exotic gamma rays from the galactic center, the galactic halo, and even from extra galaxies.

In this paper, we discuss $\mathrm{SU}(2)_{L}$ non-singlet WIMPs and the gamma ray fluxes from the galactic center due to the pair annihilation. We refer to such dark matter as electroweak-interacting massive particle (EWIMP) dark matter [15]. Due to their $\mathrm{SU}(2)_{L}$ non-singlet nature, EWIMPs have interactions with the $\mathrm{SU}(2)_{L}$ gauge bosons such as $W$ and $Z$ bosons. If EWIMPs have a vector coupling to $Z$ boson, the current bound obtained from direct dark matter searches through their spinindependent interaction is stringent [16]. This means that the EWIMP dark matter should be a Majorana fermion or a real scalar if the mass is around $1 \mathrm{TeV}$. In this paper, we consider the former case. We especially study triplet and doublet EWIMP dark matters, which are neutral components of an $\mathrm{SU}(2)_{L}$-triplet fermion whose hypercharge is zero and of a pair of $\mathrm{SU}(2)_{L}$-doublet fermions with hypercharges $\pm 1 / 2$, respectively. When the EWIMP mass is around $1 \mathrm{TeV}$, the thermal relic abundance may be consistent with the cosmological observation. 
The EWIMP is realized in the minimal supersymmetric standard model (MSSM) when the lightest SUSY particle (LSP) is the Higgsino- or Wino-like neutralino [17. Wino is the superpartner of the $\mathrm{SU}(2)_{L}$ gauge boson, and Higgsino is that of $\mathrm{SU}(2)_{L}$ doublet Higgs bosons. The thermal relic density is too low if the LSP mass is smaller than $1 \mathrm{TeV}$. However, decays of gravitino or other quasi-stable particles may produce the LSPs non-thermally so that the relic abundance is consistent with the cosmological observation. While the LSP with the mass about $1 \mathrm{TeV}$ may lead to the naturalness problem, such possibilities are discussed in the split SUSY scenario [18.

The line and continuum gamma ray fluxes from the EWIMP dark matter annihilation in the galactic center are proportional to the cross section to two photons and those to other modes whose final states fragment into $\pi^{0} \mathrm{~s}$, respectively. The leading-order cross sections in perturbation have been calculated by many authors [19. However, if the EWIMP mass is large compared to the weak gauge boson masses, usual perturbative expansion for the annihilation cross sections cannot be applied [15]. This can be seen in the violation of unitarity of the one-loop annihilation cross section into two photons. The fact comes from the degeneracy of the EWIMP and its $\mathrm{SU}(2)_{L}$ partner(s) in mass and the non-relativistic motion of the dark matter in the current universe. The transition between EWIMP and its partner pairs is induced by the $t$-channel weak gauge boson exchange. When the EWIMP mass is much larger than the weak gauge boson masses, the weak interaction behaves as a long-range force. The wave functions of EWIMP and its partner pairs are modified from plane waves at the non-relativistic limit, and the mixing between those states is enhanced. This phenomenon is related to so-called a threshold singularity, and we have to consider the effects of the long-range force on the annihilation cross sections for reliable calculation.

In this paper we work in the non-relativistic effective theory for EWIMPs. Nonrelativistic effective theories [20] are often used in calculations of the threshold productions of heavy particles, the quarkonium mass spectrums and so on [21]. In this technique, we can factorize short-distance physics, such as pair annihilation, from long-range effects on the wave functions due to the optical theorem [22]. The long-range effects are evaluated by solving the wave functions under the potential.

We found that the annihilation cross sections may be enhanced by several orders of magnitude compared to the leading-order calculation in perturbation when the EWIMP mass is larger than about $1 \mathrm{TeV}$. The mixture of the pairs of EWIMPs and 
the $\mathrm{SU}(2)_{L}$ partners can form a bound state whose binding energy is close to zero, and it contributes to the annihilation cross sections in the non-relativistic limit. The enhancement of the cross sections originates from the resonance by the bound state. Furthermore, the annihilation cross section to two photons, which is suppressed by a loop factor in perturbation, becomes comparable to those to the other modes around the resonance. As a result, the continuum and line gamma ray fluxes from the galactic center due to the EWIMP annihilation are enhanced. The indirect dark matter searches by the large ACT detectors, which have sensitivities to TeV-scale gamma rays, may be promising, if dark matter is a TeV EWIMP.

It is also found that the non-perturbative corrections to the cross sections are sizable for the triplet (doublet) EWIMP even when the EWIMP mass is about 500 (1500) GeV. Thus, the correction should be taken into account in the evaluation of the gamma ray fluxes in the MSSM, especially when the LSP is Wino-like.

This paper is organized as follows. We first summarize the properties of EWIMPs and discuss the threshold singularity in the EWIMP pair annihilation in the next section. In Section III the non-relativistic effective actions for the triplet and the doublet EWIMP pairs are derived. In Section IV the cross section formula is obtained using the optical theorem and the effective actions. While the obtained annihilation cross section to two photons in our formula is reduced to the one-loop result in the perturbative expansion, it also satisfies the unitarity bound in the limit of an infinite EWIMP mass. The one-loop cross section does not satisfies the bound.

In Section $\mathrm{V}$ some numerical results for the annihilation cross sections are presented. The fitting formulae for the annihilation cross sections are also derived from the numerical results. The resonance behaviors of the cross sections are studied using a toy model in which the electroweak Yukawa potentials are approximated by a well potential. In Section VI we evaluate the gamma ray fluxes from the EWIMP annihilation in the galactic center and discuss the sensitivities in the future experiments. In Sections $\mathrm{V}$ and VI the cross sections and the gamma ray fluxes for the Wino- and the Higgsino-like neutralinos are also evaluated in the wide range of the MSSM parameters. Section VII is devoted to summary of the paper.

\section{Properties of EWIMPs and Threshold Singularity}

In this section the mass spectrums of EWIMPs and the $\mathrm{SU}(2)_{L}$ partners and their low-energy interactions are summarized. We also discuss the threshold singularity 
in the non-relativistic EWIMP pair annihilation. The perturbative expansion of the annihilation cross sections is spoiled due to the singularity when the EWIMP mass is heavy compared to the weak gauge boson masses.

\section{Properties of EWIMPs}

The EWIMP dark matter $\tilde{\chi}^{0}$ is a neutral component of $\mathrm{SU}(2)_{L}$ multiplet(s). In this paper, we consider two cases. One is an $\mathrm{SU}(2)_{L}$ triplet fermion whose hypercharge is zero. In this case, the EWIMP is accompanied with the $\mathrm{SU}(2)_{L}$ partner, a charged Dirac fermion $\tilde{\chi}^{-}$. They are almost degenerate in mass, and the mass difference $\delta m$ is caused by the electroweak symmetry breaking. If $\delta m$ comes from the radiative correction of the gauge boson loops [23],

$$
\begin{aligned}
\delta m_{\mathrm{rad}} & =\frac{\alpha_{2} m}{4 \pi}\left[f\left(\frac{m_{W}}{m}\right)-c_{W}^{2} f\left(\frac{m_{Z}}{m}\right)-s_{W}^{2} f(0)\right] \\
f(a) & =\int_{0}^{1} d x 2(1+x) \log \left[x^{2}+(1-x) a^{2}\right]
\end{aligned}
$$

where $m$ is the EWIMP mass, $\alpha_{2}$ is the $\mathrm{SU}(2)_{L}$ gauge coupling, $m_{W}\left(m_{Z}\right)$ is the $W(Z)$ gauge boson mass, and $c_{W}\left(s_{W}\right)=\cos \theta_{W}\left(\sin \theta_{W}\right)$, where $\theta_{W}$ is the Weinberg angle. The gauge interactions of the EWIMP and its $\mathrm{SU}(2)_{L}$ partner are given by

$$
\mathcal{L}_{\text {int }}=-\frac{e}{s_{W}}\left(\overline{\tilde{\chi}^{0}} W^{\dagger} \tilde{\chi}^{-}+\text {h.c. }\right)+e \frac{c_{W}}{s_{W}} \overline{\tilde{\chi}^{-}} \not{Z} \tilde{\chi}^{-}+e \overline{\tilde{\chi}^{-}} A \tilde{\chi}^{-}
$$

where $e=\sqrt{4 \pi \alpha}$ and $\alpha$ is the fine structure constant. The mass difference $\delta m_{\text {rad }}$ is induced by the custodial $\mathrm{SU}(2)_{L}$ symmetry breaking in the gauge sector, and $\delta m_{\text {rad }} \simeq 0.18 \mathrm{GeV}$ if $m \gg m_{W}$ and $m_{Z}$. Effective higher-dimensional operators may also generate the mass difference, however it is suppressed by $m_{W}^{4} / \Lambda^{3}$, where $\Lambda$ is a new particle mass scale.

Another example of the EWIMP dark matter is a neutral component in a pair of $\mathrm{SU}(2)_{L}$ doublet fermions with the hypercharges $\pm 1 / 2$. After the symmetry breaking, two neutral mass eigenstates, $\tilde{\chi}^{0}$ and $\tilde{\chi}_{N}^{0}$, appear. The lightest one is a candidate of the EWIMP dark matter. A charged Dirac fermion $\tilde{\chi}^{-}$is also accompanied with them, and they are also degenerate in mass in the $\mathrm{SU}(2)_{L}$ symmetric limit. The mass differences among them are generated by effective operators via the electroweak symmetry breaking. Unlike the triplet EWIMP case, the mass difference is $\mathcal{O}\left(m_{W}^{2} / \Lambda\right)$ and it is not strongly suppressed by $\Lambda$. The gauge interactions of the doublet EWIMP 
dark matter and its partners are given by

$$
\begin{aligned}
\mathcal{L}_{\text {int }}= & -\frac{e}{2 s_{W}}\left(\overline{\tilde{\chi}^{0}} W^{\dagger} \tilde{\chi}^{-}-\overline{\tilde{\chi}_{N}^{0}} W^{\dagger} \tilde{\chi}^{-}+\text {h.c. }\right)-\frac{e}{s_{W} c_{W}}\left(\frac{1}{2}-c_{W}^{2}\right) \overline{\tilde{\chi}^{-}} \boldsymbol{z} \tilde{\chi}^{-} \\
& +e \overline{\tilde{\chi}^{-}} A \tilde{\chi}^{-}+\frac{e}{2 s_{W} c_{W}} \overline{\tilde{\chi}^{0}} \not \tilde{\chi}_{N}^{0} .
\end{aligned}
$$

An example of the EWIMP dark matter is the lightest neutralino in the MSSM. Neutralinos $\tilde{\chi}_{i}^{0}(i=1 \cdots 4)$ are linear combinations of the supersymmetric partners of gauge bosons and Higgs bosons, Bino $(\tilde{B})$, neutral Wino $\left(\tilde{W}^{0}\right)$ and neutral Higgsinos $\left(\tilde{H}_{1}^{0}, \tilde{H}_{2}^{0}\right)$. While those four fields have $\mathrm{SU}(2)_{L} \otimes \mathrm{U}(1)_{Y}$ invariant masses, they are mixed with each other via the electroweak symmetry breaking [17,

$$
\tilde{\chi}_{i}^{0}=Z_{i 1} \tilde{B}+Z_{i 2} \tilde{W}^{0}+Z_{i 3} \tilde{H}_{1}^{0}+Z_{i 4} \tilde{H}_{2}^{0} .
$$

Coefficients $Z_{i j}$ are determined by diagonalizing the neutralino mass matrix,

$$
M_{\tilde{\chi}^{0}}=\left(\begin{array}{cccc}
M_{1} & 0 & -m_{Z} s_{W} c_{\beta} & m_{Z} s_{W} s_{\beta} \\
0 & M_{2} & m_{Z} c_{W} c_{\beta} & -m_{Z} c_{W} s_{\beta} \\
-m_{Z} s_{W} c_{\beta} & m_{Z} c_{W} c_{\beta} & 0 & -\mu \\
m_{Z} s_{W} s_{\beta} & -m_{Z} c_{W} s_{\beta} & -\mu & 0
\end{array}\right),
$$

which is written in the $\left(\tilde{B}, \tilde{W}^{0}, \tilde{H}_{1}^{0}, \tilde{H}_{2}^{0}\right)$ basis. Here $M_{1}$ and $M_{2}$ are the Bino and Wino masses, respectively, and $\mu$ is the supersymmetric Higgsino mass. The variable $\tan \beta$ is given by the ratio of the vacuum expectation values of two Higgs fields, and $c_{\beta}=\cos \beta$ and $s_{\beta}=\sin \beta$. The lightest neutralino is Wino-like when $M_{2} \ll|\mu|, M_{1}$, and Higgsino-like when $|\mu| \ll M_{1}, M_{2}$. These two neutralinos have $\mathrm{SU}(2)_{L}$ charges and are candidates of the EWIMP dark matter; the Wino-like neutralino is a triplet EWIMP and the Higgsino-like neutralino is a doublet EWIMP.

Neutralinos are accompanied with charginos $\chi_{i}^{-}(i=1,2)$, which are linear combinations of charged Wino $\tilde{W}^{-}$and charged Higgsino $\tilde{H}^{-}=\tilde{H}_{1 L}^{-}+\tilde{H}_{2 R}^{-}$[17]. The compositions of charginos are determined by diagonalizing the chargino mass matrix,

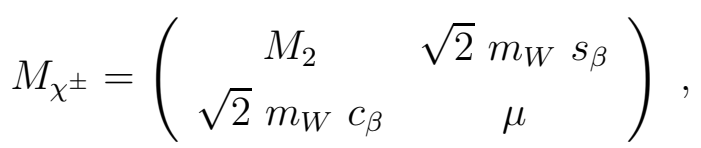

which is written in the $\left(\tilde{W}^{-}, \tilde{H}^{-}\right)$basis.

From the above matrices in Eqs. (15) and (6), the mass difference $\delta m_{\text {tree }}$ between the lightest neutralino and chargino at tree level can be calculated. If the LSP is Wino-like $\left(m_{Z}, M_{2} \ll M_{1},|\mu|\right), \delta m_{\text {tree }}$ is approximately given by

$$
\delta m_{\text {tree }} \simeq \frac{m_{Z}^{4}}{M_{1} \mu^{2}} s_{W}^{2} c_{W}^{2} \sin ^{2} 2 \beta
$$




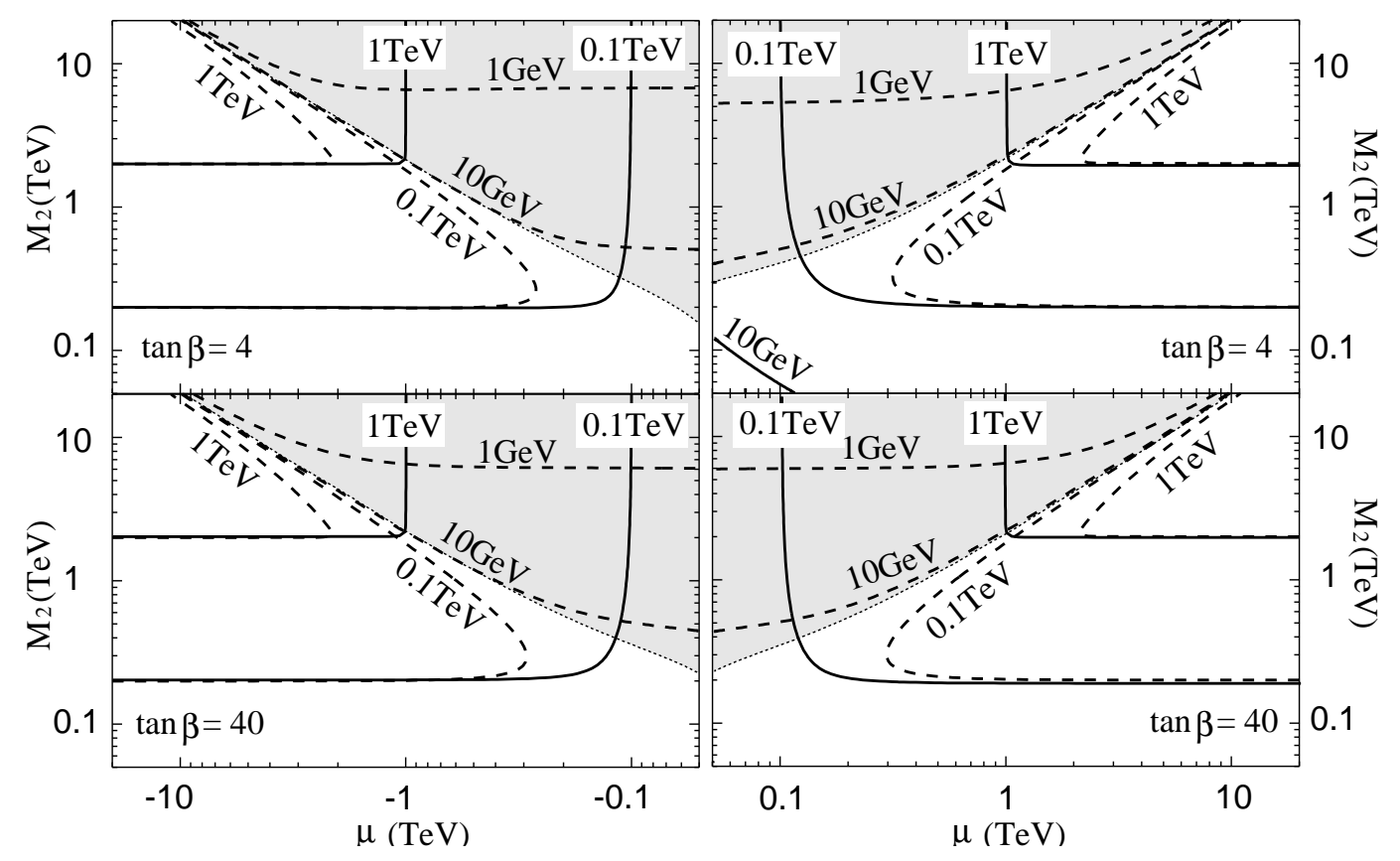

Figure 1: Contour maps of the lightest neutralino mass (solid line) and the mass difference between the lightest neutralino and chargino (dashed line) in $\left(M_{2}, \mu\right)$ planes with $\tan \beta=4$ (two top figures) and $\tan \beta=40$ (two bottom figures) in the MSSM. $M_{2}=2 M_{1}$ is assumed. Shaded areas correspond to the Higgsino-like region $\left(\left|Z_{13}\right|^{2}+\left|Z_{14}\right|^{2}>0.9\right)$.

which is suppressed by the third power of the high energy scale $M_{1} \mu^{2}$ as discussed before. Since their masses are highly degenerate at tree level, the radiative correction to the mass difference in Eq. (11) is also important.

The mass splitting for the Higgsino-like LSP in a case with $m_{Z},|\mu| \ll M_{1}, M_{2}$ is $\mathcal{O}\left(m_{Z}^{2} / m_{\text {SUSY }}\right)$ and given by

$$
\delta m \simeq \frac{1}{2} \frac{m_{Z}^{2}}{M_{2}} c_{W}^{2}(1-\sin 2 \beta)+\frac{1}{2} \frac{m_{Z}^{2}}{M_{1}} s_{W}^{2}(1+\sin 2 \beta) .
$$

The second lightest neutralino is also degenerate with the LSP and the chargino in mass, because they are in common $\mathrm{SU}(2)_{L}$ multiplets. The mass difference $\delta m_{N}$ between the LSP and the second lightest neutralino is again $\mathcal{O}\left(m_{Z}^{2} / m_{\mathrm{SUSY}}\right)$,

$$
\delta m_{N} \simeq \frac{m_{Z}^{2}}{M_{2}} c_{W}^{2}+\frac{m_{Z}^{2}}{M_{1}} s_{W}^{2}
$$

and this is $2 \times \delta m$ when $\tan \beta \gg 1$.

In Fig. 1]and Fig. 2, we show contours of the lightest neutralino mass and the mass difference between the neutralino and the lightest chargino in $\left(\mu, M_{2}\right)$ planes with $\tan \beta=4,40$. These figures are obtained by diagonalizing the mass matrices in Eqs. 

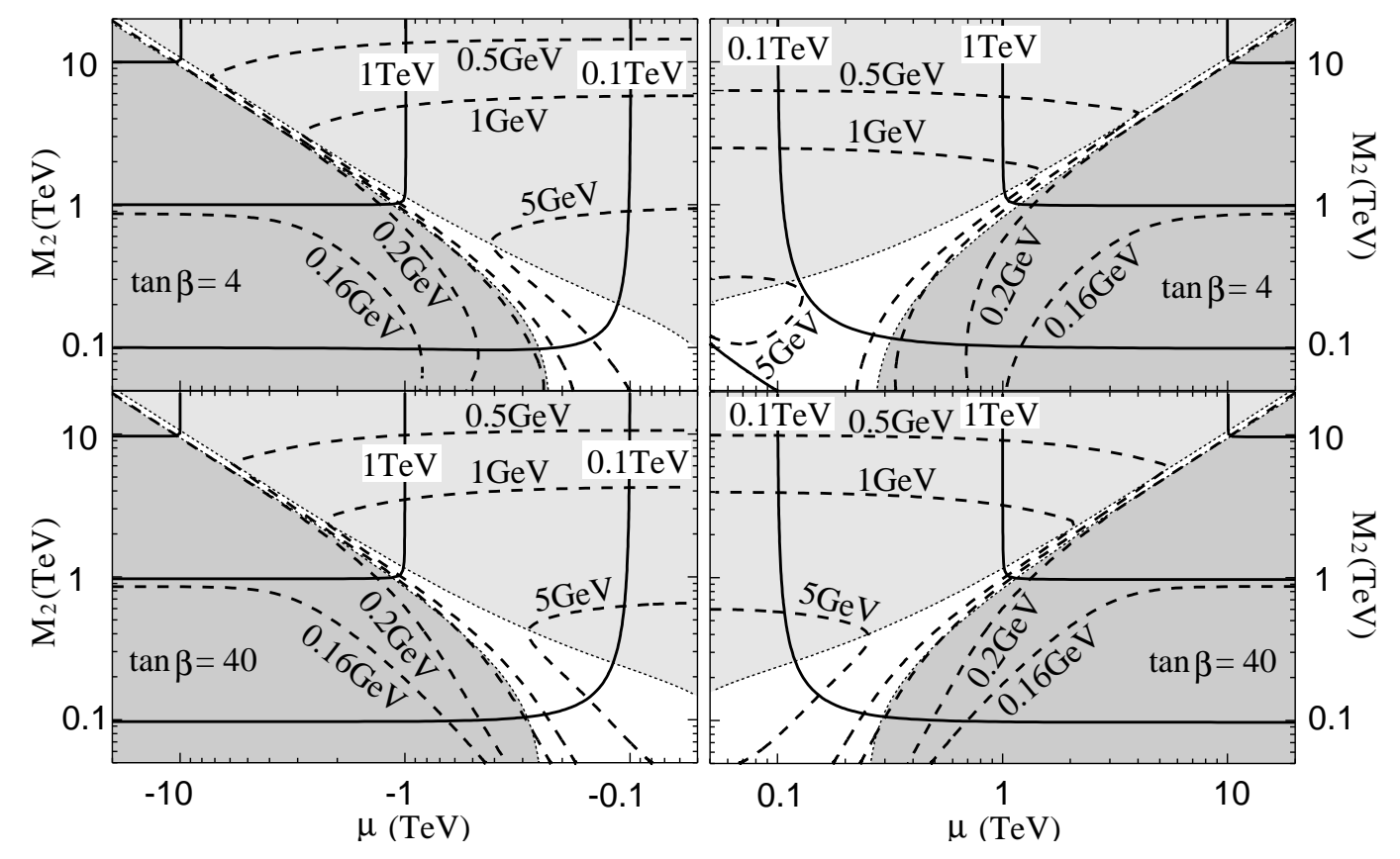

Figure 2: Contour maps of the lightest neutralino mass (solid line) and the mass difference between the lightest neutralino and chargino (dashed line) in $\left(M_{2}, \mu\right)$ planes with $\tan \beta=4$ (two top figures) and $\tan \beta=40$ (two bottom figures) in the MSSM. $M_{2}=M_{1} / 3$ is assumed. Lighter shaded areas correspond to the Higgsino-like region $\left(\left|Z_{13}\right|^{2}+\left|Z_{14}\right|^{2}>\right.$ $0.9)$, while the darker shaded areas are the Wino-like one $\left(\left|Z_{12}\right|^{2}>0.9\right)$. The radiative correction in Eq. (11) is included for depicting the contour of the mass difference in the Wino-like regions.

(15) and (6) numerically. In Fig. 11, we assume the GUT relation between the gaugino masses, $M_{2}=2 M_{1}$. In this case, the Higgsino-like neutralino (doublet EWIMP) may be the dark matter if $|\mu| \lesssim M_{1}$. The shaded areas in these figures correspond to the Higgsino-like region $\left(\left|Z_{13}\right|^{2}+\left|Z_{14}\right|^{2}>0.9\right)$, and the lightest neutralino is degenerate with the lightest chargino, especially at a large mass.

Fig. 2 is the same plots except that we assume the relation $M_{2}=M_{1} / 3$, which is predicted in the anomaly mediated supersymmetry breaking scenario [24]. In this case the Wino-like (triplet EWIMP) or the Higgsino-like (doublet EWIMP) dark matter may be realized. The lighter shaded areas (as bright as the shaded areas in Fig. (1) are the Higgsino-like region $\left(\left|Z_{13}\right|^{2}+\left|Z_{14}\right|^{2}>0.9\right)$, and the darker shaded areas are the Wino-like one $\left(\left|Z_{12}\right|^{2}>0.9\right)$. The Wino-like neutralino is highly degenerate with the lightest chargino in mass as expected.

When the triplet EWIMP mass is around $1.7 \mathrm{TeV}$, the thermal relic density of 


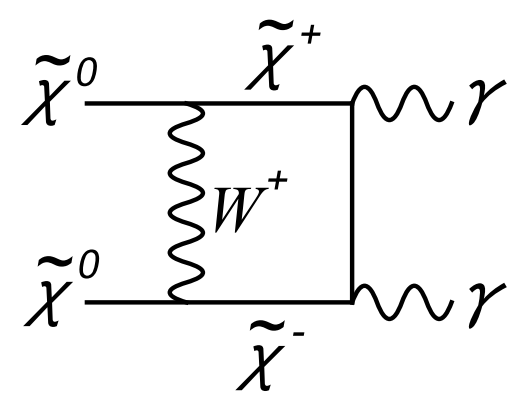

Figure 3: Dominant diagram in the Wino- or Higgsino-like neutralino annihilation to two photons at one-loop level, when the neutralino is heavy compared to the weak gauge bosons.

the dark matter is consistent with the WMAP data. In the doublet EWIMP case, the mass around $1 \mathrm{TeV}$ explains the WMAP data [25]. However, note that the dark matter in the universe may be produced thermally [26], or non-thermally [27. Therefore, we do not assume any scenarios for the dark matter relic density in this paper. Instead, we assume the dark matter forms the dark halo in our galaxy with the appropriate mass density.

\section{$\underline{\text { 2. Threshold singularity }}$}

In the calculations of the EWIMP annihilation cross sections, a threshold singularity appears due to the gauge interactions. For investigating the singularity, let us consider the EWIMP annihilation cross section to two photons $\sigma\left(\tilde{\chi}^{0} \tilde{\chi}^{0} \rightarrow \gamma \gamma\right)$ as an example. The signal line gamma ray flux from the galactic center is proportional to $\sigma\left(\tilde{\chi}^{0} \tilde{\chi}^{0} \rightarrow \gamma \gamma\right)$. This process is a radiative one, and the full one-loop non-relativistic cross section in the MSSM context has already been calculated in Ref. [19]. It is found that the cross section is suppressed only by the $W$ boson mass, not by the neutralino mass as

$$
\sigma v \sim \frac{\alpha^{2} \alpha_{2}^{2}}{m_{W}^{2}},
$$

if the neutralino is heavy and almost Wino- or Higgsino-like. The dominant diagram is shown in Fig. 3 .

On the other hand, the cross section must be bounded from above by the unitarity limit,

$$
\sigma v<\frac{4 \pi}{v m^{2}}
$$




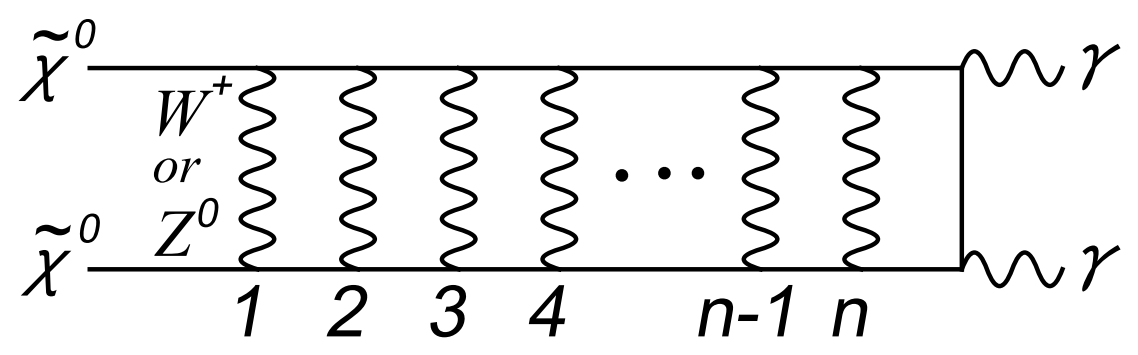

Figure 4: Dominant diagram in the Wino- or Higgsino-like neutralino annihilation at $\mathcal{O}\left(\alpha \alpha_{2}^{n}\right)$, in which $n$ weak gauge bosons are exchanged.

Thus, the one-loop cross section exceeds the bound for the extremely heavy neutralino. It means that the higher-order corrections should be included. The dominant higher-order contribution comes from the ladder diagrams. The $n$-th order $\left(\alpha_{2}^{n}\right)$ ladder diagram, in which $n$ weak gauge bosons are exchanged, is depicted in Fig. 4 , The corresponding amplitude $\mathcal{A}_{n}$ of the diagram is roughly given by

$$
\mathcal{A}_{n} \simeq \alpha\left(\frac{\alpha_{2} m}{m_{W}}\right)^{n} .
$$

When the neutralino mass $m$ is large enough, the diagrams are enhanced by a factor of $\alpha_{2} m / m_{W}$ for each weak gauge boson exchange. The higher-order loop diagrams become more and more important when $\alpha_{2} m \gtrsim m_{W}$.

Enhancement of ladder diagrams in non-relativistic limits is related to a threshold singularity. Recall that a threshold singularity appears in the non-relativistic $\mu^{+} \mu^{-}$ pair annihilation cross section. When the relative velocity $v$ of the muon pair is smaller than $\alpha$, the amplitude of the $n$-order ladder diagram, in which $n$ photons are exchanged between the muon pair, is proportional to $\alpha(\alpha / v)^{n}$, and the perturbative expansion by $\alpha$ breaks down. The internal muons are close to non-relativistic onshell states. The muon and photon propagaters are proportional to $1 / v^{2}$ and each loop integration gives $\alpha v^{5}$. Thus, the diagrams are enhanced by $\alpha / v$ for each photon exchange. This is because the kinetic energy of muon pair, $m_{\mu} v^{2} / 4$, is smaller than the Coulomb potential energy, $\alpha^{2} m_{\mu}$, and the wave function of the incident particles is deformed from plane waves. We need to systematically resum the ladder diagrams or to use the wave function under the Coulomb potential in order to get the precise annihilation cross section.

In the non-relativistic EWIMP pair annihilation, the sub-diagram corresponding to the process $\tilde{\chi}^{0} \tilde{\chi}^{0} \rightarrow \tilde{\chi}^{+} \tilde{\chi}^{-}$in each ladder diagram is very close to the threshold 
when the mass difference $\delta m$ is negligible. In this case, the spatial momentums for EWIMPs and the $\mathrm{SU}(2)_{L}$ partners in the internal lines are regularized by the weak gauge boson masses. Their propagaters and the weak gauge boson ones behave as $m / m_{W}^{2}$ and $1 / m_{W}^{2}$, respectively, and the loop integration gives $\alpha_{2} m_{W}^{5} / m$. Thus, the diagrams are enhanced by $\alpha_{2} m / m_{W}$ for each weak gauge boson exchange, as shown above. This implies that when $\alpha_{2} m \gtrsim m_{W}$, the weak interaction becomes a long-range force and the wave function is significantly modified inside the Yukawa potentials induced by the weak gauge boson exchanges in the non-relativistic limit. In the following sections, we will introduce a systematic method to derive the annihilation cross sections in the threshold singularity region by using the non-relativistic action.

The elastic scattering cross section of dark matter with nucleon is important for the direct search for dark matter [28. If the dark matter is an EWIMP and much heavier than the weak gauge boson masses, the one-loop correction to the cross section is suppressed only by the weak gauge boson masses and it may be dominant over the tree-level contribution [29]. However, the perturbative expansion is still reliable, unlike the case of the annihilation cross sections. This is because the reduced mass in the EWIMP and nucleon two-body system is not heavy enough for non-perturbative corrections to be sizable.

\section{Two-body State Effective Action}

In this section we derive the effective actions for the non-relativistic two-body states including pairs of EWIMPs and the $\mathrm{SU}(2)_{L}$ partners. The action is derived by following steps. (i) We integrate out all fields except EWIMPs and the $\mathrm{SU}(2)_{L}$ partners such as the lightest neutralino and chargino in the MSSM action. (ii) The non-relativistic action $\mathcal{S}_{\mathrm{NR}}$ is obtained by integrating out large momentum modes of EWIMPs and the $\mathrm{SU}(2)_{L}$ partners. The action includes the effect of the EWIMP annihilation as the absorptive parts. (iii) The action $\mathcal{S}_{\mathrm{NR}}$ is expanded by the velocities of EWIMPs. (iv) At last, we introduce auxiliary fields to the action $\mathcal{S}_{\mathrm{NR}}$, which represent two-body states of EWIMPs and the $\mathrm{SU}(2)_{L}$ partners. The two-body state

effective action $\mathcal{S}^{(I I)}$ is obtained by integrating out all fields except those auxiliary fields in the non-relativistic action $\mathcal{S}_{\mathrm{NR}}$.

In the following, we derive the two-body state effective action for the triplet EWIMP (Wino-like neutralino). For the doublet EWIMP (Higgsino-like neutralino), 
only the final result is presented, because the derivation is essentially the same as that of the triplet one.

\section{Integrating out all fields except $\tilde{\chi}^{0}$ and $\tilde{\chi}^{-}$}

Relevant interactions to the annihilation cross section for the triplet EWIMP are the gauge interaction in Eq. (2). In the MSSM, the lightest neutralino and chargino have other interactions with sfermions and Higgs bosons. However, the contributions to the annihilation cross sections are suppressed by the sfermion masses or the gaugino-Higgsino mixing. We assume that these contributions are small, and neglect them.

After integrating out the gauge bosons, the effective action for the triplet EWIMP and its charged $\mathrm{SU}(2)_{L}$ partner becomes

$$
\begin{array}{r}
\mathcal{S}_{\text {eff }}=\int d^{4} x\left[\frac{1}{2} \overline{\tilde{\chi}^{0}}(i \not \partial-m) \tilde{\chi}^{0}+\overline{\tilde{\chi}^{-}}\left(i \not \partial-m_{c}\right) \tilde{\chi}^{-}\right]+\mathcal{S}_{\text {int }}\left[\tilde{\chi}^{0}, \tilde{\chi}^{-}\right] \\
\mathcal{S}_{\mathrm{int}}=2 i \pi \alpha \int d^{4} x_{1} d^{4} x_{2}\left[\frac{2}{s_{W}^{2}} j_{W}^{\mu \dagger}\left(x_{1}\right) G_{\mu \nu}^{(W)}\left(x_{1}-x_{2}\right) j_{W}^{\nu}\left(x_{2}\right)\right. \\
\left.+j_{\chi^{-}}^{\mu}\left(x_{1}\right)\left\{G_{\mu \nu}^{(\gamma)}\left(x_{1}-x_{2}\right)+\frac{c_{W}^{2}}{s_{W}^{2}} G_{\mu \nu}^{(Z)}\left(x_{1}-x_{2}\right)\right\} j_{\chi^{-}}^{\mu}\left(x_{2}\right)\right],
\end{array}
$$

where the parameter $m_{c}$ is the mass of the charged $\mathrm{SU}(2)_{L}$ partner. The functions $G_{\mu \nu}^{(W)}(x), G_{\mu \nu}^{(Z)}(x)$ and $G_{\mu \nu}^{(\gamma)}(x)$ are the Feynman propagaters of the $W, Z$ bosons and photon, respectively. The currents $j_{W}^{\mu}(x)$ and $j_{\chi^{-}}^{\mu}(x)$ are defined as

$$
j_{W}^{\mu}(x)=\overline{\tilde{\chi}^{0}}(x) \gamma^{\mu} \tilde{\chi}^{-}(x), \quad j_{\chi^{-}}^{\mu}(x)=\overline{\tilde{\chi}}^{-}(x) \gamma^{\mu} \tilde{\chi}^{-}(x)
$$

Here we include the effects of the non-vanishing mass difference between the EWIMP and its $\mathrm{SU}(2)_{L}$ partner, $\delta m\left(\equiv m_{c}-m\right)$, which comes from the electroweak symmetry breaking. The non-vanishing $\delta m$ gives sizable effects on the annihilation cross sections when $\delta m$ is not negligible compared with $\alpha_{2} m_{W}$ as will be shown in Sec. V. On the other hand, the $\mathrm{SU}(2)_{L} \otimes \mathrm{U}(1)_{Y}$ breaking in the gauge interactions gives at most corrections up to $O\left(m_{W}^{2} / \Lambda^{2}\right)$ to the cross sections, and they can be ignored as far as $m_{W} \ll \Lambda$.

2. Integrating out large momentum modes of $\tilde{\chi}^{0}$ and $\tilde{\chi}^{-}$

We now derive the action which describes the non-relativistic motion of the EWIMP and its $\mathrm{SU}(2)_{L}$ partner. Namely we integrate out the large momentum 
modes of these particles in $\mathcal{S}_{\text {eff }}$. We divide the fields $\tilde{\chi}^{0}$ and $\tilde{\chi}^{-}$into two parts, the non-relativistic part and the other,

$$
\begin{aligned}
\tilde{\chi}^{0}(x) & =\tilde{\chi}_{\mathrm{NR}}^{0}(x)+\delta \tilde{\chi}^{0}(x) \\
\tilde{\chi}_{\mathrm{NR}}^{0}(x) & =\int_{[\mathrm{NR}]} \frac{d^{4} p}{(2 \pi)^{4}} \phi^{0}(p) e^{-i p x}, \quad \delta \tilde{\chi}^{0}(x)=\int_{[\overline{\mathrm{NR}}]} \frac{d^{4} p}{(2 \pi)^{4}} \phi^{0}(p) e^{-i p x},
\end{aligned}
$$

where the $\phi^{0}(p)$ is the Fourier coefficient of the EWIMP field. The region of the integration $[\mathrm{NR}]$ is defined roughly by $[\mathrm{NR}]=\left\{\left(p^{0}, \vec{p}\right) \mid p^{0}= \pm m+\mathcal{O}\left(m|\vec{v}|^{2}\right), \vec{p}=\right.$ $\mathcal{O}(m \vec{v}),|\vec{v}| \ll 1\}$, and $[\overline{\mathrm{NR}}]$ means the complementary set of $[\mathrm{NR}]$. The $\mathrm{SU}(2)_{L}$ partner field $\tilde{\chi}^{-}$is also divided into $\tilde{\chi}_{\mathrm{NR}}^{-}$and $\delta \tilde{\chi}^{-}$in the same way. After integrating out large momentum modes $\delta \tilde{\chi}^{0}$ and $\delta \tilde{\chi}^{-}$in the action $\mathcal{S}_{\text {eff }}$, the non-relativistic effective action $\mathcal{S}_{\mathrm{NR}}$ is obtained as

$$
\begin{aligned}
\mathcal{S}_{\mathrm{NR}}= & \int d^{4} x\left[\frac{1}{2} \overline{\tilde{\chi}}_{\mathrm{NR}}^{0}(i \not \partial-m) \tilde{\chi}_{\mathrm{NR}}^{0}+\overline{\tilde{\chi}}^{-}{ }_{\mathrm{NR}}\left(i \not \partial-m_{c}\right) \tilde{\chi}_{\mathrm{NR}}^{-}\right] \\
& +\mathcal{S}_{\mathrm{int}}\left[\tilde{\chi}_{\mathrm{NR}}^{0}, \tilde{\chi}_{\mathrm{NR}}^{-}\right]+\delta \mathcal{S}\left[\tilde{\chi}_{\mathrm{NR}}^{0}, \tilde{\chi}_{\mathrm{NR}}^{-}\right] .
\end{aligned}
$$

All effective interactions induced from the integration by $\delta \tilde{\chi}^{0}$ and $\delta \tilde{\chi}^{-}$are included in $\delta \mathcal{S}$. Though they are suppressed by the EWIMP mass in comparison with interactions in $\mathcal{S}_{\text {int }}$, they give leading contributions to the imaginary part (absorptive part) of the non-relativistic action. The action is further simplified in the next step.

\section{Non-relativistic expansion of the action $\mathcal{S}_{\mathrm{NR}}$}

Here, we expand the action $\mathcal{S}_{\mathrm{NR}}$ by the velocity of the EWIMP. For the expansion, it is convenient to use two-components spinor fields $\zeta, \eta$ and $\xi$ instead of $\tilde{\chi}_{\mathrm{NR}}^{0}$ and $\tilde{\chi}_{\mathrm{NR}}^{-} \cdot$ These spinor fields are defined by

$$
\tilde{\chi}_{\mathrm{NR}}^{0}=\left(\begin{array}{c}
e^{-i m t} \zeta+i e^{i m t} \frac{\vec{\nabla} \cdot \vec{\sigma}}{2 m} \zeta^{c} \\
e^{i m t} \zeta^{c}-i e^{-i m t} \frac{\vec{\nabla} \cdot \vec{\sigma}}{2 m} \zeta
\end{array}\right), \quad \tilde{\chi}_{\mathrm{NR}}^{-}=\left(\begin{array}{c}
e^{-i m t} \eta+i e^{i m t} \frac{\vec{\nabla} \cdot \vec{\sigma}}{2 m} \xi \\
e^{i m t} \xi-i e^{-i m t} \frac{\vec{\nabla} \cdot \vec{\sigma}}{2 m} \eta
\end{array}\right) .
$$

The spinor $\zeta^{c}$ is the charge conjugation of $\zeta, \zeta^{c}=-i \sigma^{2} \zeta^{*}$, where $\sigma_{2}$ is the Pauli matrix. Spinors $\zeta$ and $\eta$ annihilate one $\tilde{\chi}^{0}$ and one $\tilde{\chi}^{-}$, respectively, while $\xi$ creates one $\tilde{\chi}^{+}$. 
The non-relativistic action $\mathcal{S}_{\mathrm{NR}}$ is systematically expanded by the velocity of the two-components spinor fields. The kinetic terms in Eq. (17) become

$$
\begin{aligned}
\left.S_{\mathrm{NR}}\right|_{\text {kinetic terms }}=\int d^{4} x\left[\zeta^{\dagger}\left(i \partial_{t}+\frac{\nabla^{2}}{2 m}\right) \zeta\right. & +\eta^{\dagger}\left(i \partial_{t}-\delta m+\frac{\nabla^{2}}{2 m}\right) \eta \\
& \left.+\xi^{\dagger}\left(i \partial_{t}+\delta m-\frac{\nabla^{2}}{2 m}\right) \xi\right] .
\end{aligned}
$$

The interactions in $\mathcal{S}_{\text {int }}$ of Eq. (17) are reduced as

$$
\begin{array}{r}
\mathcal{S}_{\mathrm{int}}=\int d^{4} x d^{3} y\left[\quad \frac{\alpha}{2|\vec{x}-\vec{y}|}\left(1+\frac{c_{W}^{2}}{s_{W}^{2}} e^{-m_{Z}|\vec{x}-\vec{y}|}\right) \eta^{\dagger}(x) \xi\left(\vec{y}, x^{0}\right) \times \xi^{\dagger}\left(\vec{y}, x^{0}\right) \eta(x)\right. \\
\left.+\frac{\alpha_{2} e^{-m_{W}|\vec{x}-\vec{y}|}}{2|\vec{x}-\vec{y}|}\left\{\zeta^{\dagger}(x) \zeta^{c}\left(\vec{y}, x^{0}\right) \times \xi^{\dagger}\left(\vec{y}, x^{0}\right) \eta(x)+\text { h.c. }\right\}\right],(20)
\end{array}
$$

where we keep terms which dictate the transitions between states with both spin and isospin singlet. The EWIMP $S$-wave state is spin-singlet due to the Majorana nature, and only those terms are kept to calculate the EWIMP annihilation cross sections at the non-relativistic limit. The first term in the parenthesis describes the Coulomb force and the force by one $Z$-boson exchange between $\tilde{\chi}^{+}$and $\tilde{\chi}^{-}$. The second term is for the transition between $\tilde{\chi}^{0} \tilde{\chi}^{0}$ and $\tilde{\chi}^{+} \tilde{\chi}^{-}$by one $W$ boson exchange.

The imaginary part (absorptive part) of the action $\mathcal{S}_{\mathrm{NR}}$ is in $\delta \mathcal{S}$ in Eq. (17). It comes from following box diagrams; (a) transitions from $\tilde{\chi}^{+} \tilde{\chi}^{-}$to $\tilde{\chi}^{+} \tilde{\chi}^{-}$with intermediate $W^{+} W^{-}, Z^{0} Z^{0}, \gamma Z$ and $\gamma \gamma$ states, (b) a transition from $\tilde{\chi}^{0} \tilde{\chi}^{0}$ to $\tilde{\chi}^{0} \tilde{\chi}^{0}$ with an intermediate $W^{+} W^{-}$state and (c) a transition from $\tilde{\chi}^{0} \tilde{\chi}^{0}$ to $\tilde{\chi}^{+} \tilde{\chi}^{-}$with an intermediate $W^{+} W^{-}$state. These effective interactions are simplified in the nonrelativistic expansion as

$$
\begin{aligned}
\delta \mathcal{S}=\frac{i \pi \alpha_{2}^{2}}{m^{2}} \int d^{4} x[ & \left(\frac{1}{2}+c_{W}^{4}+2 s_{W}^{2} c_{W}^{2}+s_{W}^{4}\right) \eta^{\dagger} \xi \cdot \xi^{\dagger} \eta \\
& \left.+2 \zeta^{\dagger} \zeta^{c} \cdot \zeta^{c \dagger} \zeta+\frac{1}{2}\left(\zeta^{\dagger} \zeta^{c} \cdot \xi^{\dagger} \eta+\text { h.c. }\right)\right] .
\end{aligned}
$$

Here, we assume that the EWIMP mass $m$ is much heavier than the weak gauge boson masses, $m \gg m_{W}, m_{Z}$. The first term of the right-hand side in Eq. (21) corresponds to the box diagrams (a). Each term in the parenthesis comes from the diagrams with intermediate $W^{+} W^{-}, Z^{0} Z^{0}, \gamma Z$ and $\gamma \gamma$ states, respectively. The second and third terms correspond to the diagrams of (b) and (c), respectively.

4. Two-body state effective action 
The non-relativistic action for the triplet EWIMP is now given by Eqs. (19- 21). We now introduce auxiliary fields $\sigma_{N}$ and $\sigma_{C}$, which describe the two-body states $\tilde{\chi}^{0} \tilde{\chi}^{0}$ and $\tilde{\chi}^{+} \tilde{\chi}^{-}$with both spin and isospin singlet, respectively. We thus insert identities

$$
\begin{aligned}
1 & =\int \mathcal{D} \sigma_{N} \mathcal{D} s_{N}^{\dagger} \exp \left[\frac{i}{2} \int d^{4} x d^{3} y \sigma_{N}(x, \vec{y})\left\{s_{N}^{\dagger}(\vec{y}, x)-\frac{1}{2} \zeta^{\dagger}(x) \zeta^{c}\left(\vec{y}, x^{0}\right)\right\}\right], \\
1 & =\int \mathcal{D} \sigma_{C} \mathcal{D} s_{C}^{\dagger} \exp \left[\frac{i}{2} \int d^{4} x d^{3} y \sigma_{C}(x, \vec{y})\left\{s_{C}^{\dagger}(\vec{y}, x)-\eta^{\dagger}(x) \xi\left(\vec{y}, x^{0}\right)\right\}\right],
\end{aligned}
$$

and their conjugates into the partition function described by the non-relativistic action. After integrating out $\eta, \xi, \zeta, s_{N}, s_{C}$ and their conjugates, the two-body state effective action $\mathcal{S}^{(I I)}$ is obtained as

$$
\mathcal{S}^{(I I)}=\int d^{4} x d^{3} r \Phi^{\dagger}(x, \vec{r})\left\{\left(i \partial_{x^{0}}+\frac{\nabla_{x}^{2}}{4 m}+\frac{\nabla_{r}^{2}}{m}\right)-\mathbf{V}(\vec{r})+2 i \boldsymbol{\Gamma} \delta(\vec{r})\right\} \Phi(x, \vec{r}),
$$

where the argument $x$ denotes the center of mass coordinate in the two-body system and $\vec{r}$ is the relative coordinate. The two-components two-body state field $\Phi(x, \vec{r})$ is defined by

$$
\Phi(x, \vec{r})=\left(\begin{array}{c}
\phi_{C}(x, \vec{r}) \\
\phi_{N}(x, \vec{r})
\end{array}\right)=(-\mathbf{V}(r)+2 i \boldsymbol{\Gamma} \delta(\vec{r}))^{-1}\left(\begin{array}{c}
\sigma_{C}(x, \vec{r}) \\
\sigma_{N}(x, \vec{r})
\end{array}\right) .
$$

The components, $\phi_{N}$ and $\phi_{C}$, describe pairs of the EWIMPs and the $\mathrm{SU}(2)_{L}$ partners, respectively. These can be also written as

$$
\begin{aligned}
\phi_{C}(x, \vec{r}) & =\frac{1}{\sqrt{2}} \xi^{\dagger}\left(\vec{x}-\vec{r} / 2, x^{0}\right) \eta\left(\vec{x}+\vec{r} / 2, x^{0}\right), \\
\phi_{N}(x, \vec{r}) & =\frac{1}{2} \zeta^{c^{\dagger}}\left(\vec{x}-\vec{r} / 2, x^{0}\right) \zeta\left(\vec{x}+\vec{r} / 2, x^{0}\right),
\end{aligned}
$$

by using $\zeta, \eta$ and $\xi$ fields. The difference between the normalizations of $\phi_{C}$ in Eq. (25) and $\phi_{N}$ in Eq. (26) comes from the fact that $\phi_{N}$ describes a pair of the identical Majorana fermions.

The electroweak potential $\mathbf{V}(r)$ in Eq. (23) is given by

$$
\mathbf{V}(r)=\left(\begin{array}{cc}
2 \delta m-\frac{\alpha}{r}-\alpha_{2} c_{W}^{2} \frac{e^{-m_{Z^{r}}}}{r} & -\sqrt{2} \alpha_{2} \frac{e^{-m_{W} r}}{r} \\
-\sqrt{2} \alpha_{2} \frac{e^{-m_{W} r}}{r} & 0
\end{array}\right) .
$$


The absorptive (imaginary) part $\boldsymbol{\Gamma}$ is decomposed as $\boldsymbol{\Gamma}=\boldsymbol{\Gamma}_{W^{+} W^{-}}+\boldsymbol{\Gamma}_{Z^{0} Z^{0}}+\boldsymbol{\Gamma}_{\gamma Z^{0}}+\boldsymbol{\Gamma}_{\gamma \gamma}$ where each component is

$$
\begin{aligned}
\boldsymbol{\Gamma}_{W^{+} W^{-}}=\frac{\pi \alpha_{2}^{2}}{4 m^{2}}\left(\begin{array}{cc}
2 & \sqrt{2} \\
\sqrt{2} & 4
\end{array}\right), \quad \boldsymbol{\Gamma}_{Z^{0} Z^{0}}=\frac{\pi \alpha_{2}^{2}}{m^{2}}\left(\begin{array}{cc}
c_{W}^{4} & 0 \\
0 & 0
\end{array}\right), \\
\boldsymbol{\Gamma}_{\gamma Z^{0}}=\frac{\pi \alpha \alpha_{2}}{m^{2}}\left(\begin{array}{cc}
2 c_{W}^{2} & 0 \\
0 & 0
\end{array}\right), \quad \boldsymbol{\Gamma}_{\gamma \gamma}=\frac{\pi \alpha^{2}}{m^{2}}\left(\begin{array}{ll}
1 & 0 \\
0 & 0
\end{array}\right) .
\end{aligned}
$$

The two-body state effective action Eq. (23) is the final result of this section.

In the case of the doublet EWIMP, the derivation of the effective action is parallel to that of the triplet one. The EWIMP is accompanied with the neutral $\mathrm{SU}(2)_{L}$ partner in addition to the charged one. The two-body effective action becomes $3 \times 3$ matrix form, and the two-body state field $\Phi(x, \vec{r})$ has three components,

$$
\Phi(x, \vec{r})=\left(\begin{array}{c}
\phi_{C}(x, \vec{r}) \\
\phi_{N}(x, \vec{r}) \\
\phi_{N_{2}}(x, \vec{r})
\end{array}\right),
$$

where $\phi_{N}, \phi_{C}$ and $\phi_{N_{2}}$ describe pairs of the EWIMPs, the charged and the neutral partners, respectively. The electroweak potential is

$$
\mathbf{V}(r)=\left(\begin{array}{ccc}
2 \delta m-\frac{\alpha}{r}-\frac{\alpha_{2}\left(1-2 c_{W}^{2}\right)^{2}}{4 c_{W}^{2}} \frac{e^{-m_{Z} r}}{r} & -\frac{\sqrt{2} \alpha_{2} e^{-m_{W} r}}{4 r} & -\frac{\sqrt{2} \alpha_{2} e^{-m_{W} r}}{4 r} \\
-\frac{\sqrt{2} \alpha_{2} e^{-m_{W} r}}{4 r} & 0 & -\frac{\alpha_{2} e^{-m_{Z} r}}{4 c_{W}^{2} r} \\
-\frac{\sqrt{2} \alpha_{2} e^{-m_{W} r}}{4 r} & -\frac{\alpha_{2} e^{-m_{Z} r}}{4 c_{W}^{2} r} & 2 \delta m_{N}
\end{array}\right),
$$

where $\delta m_{N}$ is the mass difference between the EWIMP and its neutral SU(2) $L$ partner. The absorptive part $\boldsymbol{\Gamma}$ in this case is given by $\boldsymbol{\Gamma}=\boldsymbol{\Gamma}_{W^{+} W^{-}}+\boldsymbol{\Gamma}_{Z^{0} Z^{0}}+\boldsymbol{\Gamma}_{\gamma Z^{0}}+\boldsymbol{\Gamma}_{\gamma \gamma}$, where

$$
\begin{aligned}
\boldsymbol{\Gamma}_{W^{+} W^{-}} & =\frac{\pi \alpha_{2}^{2}}{64 m^{2}}\left(\begin{array}{ccc}
8 & \sqrt{2} & \sqrt{2} \\
\sqrt{2} & 4 & 4 \\
\sqrt{2} & 4 & 4
\end{array}\right), \\
\boldsymbol{\Gamma}_{Z^{0} Z^{0}} & =\frac{\pi \alpha_{2}^{2}}{64 c_{W}^{4} m^{2}}\left(\begin{array}{ccc}
4\left(1-2 s_{W}^{2}\right)^{4} & \sqrt{2}\left(1-2 s_{W}^{2}\right)^{2} & \sqrt{2}\left(1-2 s_{W}^{2}\right)^{2} \\
\sqrt{2}\left(1-2 s_{W}^{2}\right)^{2} & 2 & 2 \\
\sqrt{2}\left(1-2 s_{W}^{2}\right)^{2} & 2 & 2
\end{array}\right), \\
\boldsymbol{\Gamma}_{\gamma Z^{0}} & =\frac{\pi \alpha \alpha_{2}}{2 c_{W}^{2} m^{2}}\left(\begin{array}{ccc}
\left(1-2 s_{W}^{2}\right)^{2} & 0 & 0 \\
0 & 0 & 0 \\
0 & 0 & 0
\end{array}\right), \quad \boldsymbol{\Gamma}_{\gamma \gamma}=\frac{\pi \alpha^{2}}{m^{2}}\left(\begin{array}{ccc}
1 & 0 & 0 \\
0 & 0 & 0 \\
0 & 0 & 0
\end{array}\right) .(31
\end{aligned}
$$




\section{Optical Theorem and Annihilation Cross Sections}

We now derive the EWIMP pair annihilation cross sections by using the twobody state effective actions in Eq. (23) . Using the formula, we also show that the one-loop cross section of $\tilde{\chi}^{0} \tilde{\chi}^{0} \rightarrow 2 \gamma$ is reproduced in the perturbative expression while the cross section in a limit of $m \rightarrow \infty$ satisfies the unitarity bound.

\section{Annihilation cross section formula}

Due to the optical theorem, the total EWIMP pair annihilation cross section is written by the imaginary part of the amplitude as

$$
\sqrt{s^{2}-4 m^{2} s} \sigma=\Im\left[\mathcal{M}_{i i}\right]
$$

where $\sqrt{s}$ is the center of mass energy, and $\mathcal{M}_{i i}$ is the invariant amplitude of the process, $\tilde{\chi}^{0} \tilde{\chi}^{0} \rightarrow \tilde{\chi}^{0} \tilde{\chi}^{0}$. When the incident EWIMPs are highly non-relativistic, the relevant initial state for the pair annihilation is only the spin-singlet $S$-wave state as mentioned before. We thus project the above equation (32) to the ${ }^{1} S_{0}$ state by using the projection operator $\int d^{3} P d k\left|\vec{P}, k,{ }^{1} S_{0} ; N\right\rangle\left\langle\vec{P}, k,{ }^{1} S_{0} ; N\right|$,

$$
\begin{aligned}
& \left|\vec{P}, k,{ }^{1} S_{0} ; N\right\rangle=\frac{k}{4 \sqrt{\pi}} \int d \Omega_{k}\left[a_{+}^{\dagger}(\vec{P} / 2+\vec{k}) a_{-}^{\dagger}(\vec{P} / 2-\vec{k})\right. \\
& \left.-a_{-}^{\dagger}(\vec{P} / 2+\vec{k}) a_{+}^{\dagger}(\vec{P} / 2-\vec{k})\right]|0\rangle,
\end{aligned}
$$

where $\vec{P}$ is the total momentum and $\vec{k}$ is the relative momentum in the EWIMP twobody system. The variable $k$ is $k=|\vec{k}|$. The state vector $\left|\vec{P}, k,{ }^{1} S_{0}\right\rangle$ is normalized as $\left\langle\vec{P}^{\prime}, k^{\prime},{ }^{1} S_{0} ; N \mid \vec{P}, k,{ }^{1} S_{0} ; N\right\rangle=\delta\left(\vec{P}-\vec{P}^{\prime}\right) \delta\left(k-k^{\prime}\right)$. The operator $a_{ \pm}^{\dagger}(\vec{k})$ creates one $\tilde{\chi}^{0}$ with the momentum $\vec{k}$ and the spin $\pm 1 / 2$, and satisfies the anti-commutation relation $\left\{a_{s}(\vec{p}), a_{s^{\prime}}^{\dagger}\left(\vec{p}^{\prime}\right)\right\}=\delta\left(\vec{p}-\vec{p}^{\prime}\right) \delta_{s s^{\prime}}$.

If the $\tilde{\chi}^{+} \tilde{\chi}^{-}$annihilation cross section is considered in a spin singlet and $S$-wave system, the projection operator $\int d^{3} P d k\left|\vec{P}, k,{ }^{1} S_{0} ; C\right\rangle\left\langle\vec{P}, k,{ }^{1} S_{0} ; C\right|$ is used instead of one in Eq. (33), where

$$
\begin{aligned}
& \left|\vec{P}, k,{ }^{1} S_{0} ; C\right\rangle=\frac{k}{2 \sqrt{2 \pi}} \int d \Omega_{k}\left[b_{+}^{\dagger}(\vec{P} / 2+\vec{k}) d_{-}^{\dagger}(\vec{P} / 2-\vec{k})\right. \\
& \left.-b_{-}^{\dagger}(\vec{P} / 2+\vec{k}) d_{+}^{\dagger}(\vec{P} / 2-\vec{k})\right]|0\rangle .
\end{aligned}
$$

The operators $b_{ \pm}^{\dagger}(\vec{k})$ and $d_{ \pm}^{\dagger}(\vec{k})$ create one $\tilde{\chi}^{-}$and one $\tilde{\chi}^{+}$with the momentum $\vec{k}$ and the spin $\pm 1 / 2$, respectively. They satisfy the anti-commutation relations $\left\{b_{s}(\vec{p}), b_{s^{\prime}}^{\dagger}\left(\vec{p}^{\prime}\right)\right\}=\left\{d_{s}(\vec{p}), d_{s^{\prime}}^{\dagger}\left(\vec{p}^{\prime}\right)\right\}=\delta\left(\vec{p}-\vec{p}^{\prime}\right) \delta_{s s^{\prime}}$. 
After the projection, the $S$-wave cross sections $\sigma_{i}^{(S)}(i=1,2)$ become

$$
\sigma_{i}^{(S)}=c_{i} \frac{32 \pi^{5}}{m^{2} v^{3}} \Im\left[\mathcal{M}_{i}^{(S)}(v)\right]
$$

where $v$ is the relative velocity between incident particles. The cross section $\sigma_{i}^{(S)}$ is for $\tilde{\chi}^{0} \tilde{\chi}^{0}$ annihilation $(i=2)$ and $\tilde{\chi}^{+} \tilde{\chi}^{-}$annihilation $(i=1)$. The coefficient $c_{i}$ is given by $c_{1}=1$ and $c_{2}=2 . \mathcal{M}_{i}^{(S)}(v)$ is the invariant amplitude from the ${ }^{1} S_{0}$ state to the ${ }^{1} S_{0}$ state. We use the non-relativistic approximation as $s \simeq 4 m^{2}+m^{2} v^{2}$ for deriving the cross sections.

The invariant amplitude $\mathcal{M}_{i}^{(S)}(v)$ is obtained from the $S$-wave Green function of the two-body state field $\Phi$. From the two-body state effective action in Eq. (23), the Schwinger-Dyson equation (equation of motion for the Green function) is derived as

$$
\begin{aligned}
\left(i \partial_{x^{0}}+\frac{\nabla_{x}^{2}}{4 m}+\frac{\nabla_{r}^{2}}{m}-\mathbf{V}(r)+i \boldsymbol{\Gamma} \frac{\delta(r)}{2 \pi r^{2}}\right)\left\langle 0\left|T \Phi(x, \vec{r}) \Phi^{\dagger}\left(y, \vec{r}^{\prime}\right)\right| 0\right\rangle & \\
= & i \delta^{(4)}(x-y) \delta^{(3)}\left(\vec{r}-\vec{r}^{\prime}\right) .
\end{aligned}
$$

The potential $\mathbf{V}(r)$ and the absorptive part $\boldsymbol{\Gamma}$ are defined in Eqs. (27) and (28) for the triplet case, and in Eqs. (301) and (31) for the doublet case. Since the potential depends on only $r$, the Green function can be expanded by the Legendre Polynomials as

$$
\left\langle 0\left|T \Phi(x, \vec{r}) \Phi^{\dagger}\left(y, \vec{r}^{\prime}\right)\right| 0\right\rangle=\int \frac{d^{4} P}{(2 \pi)^{4}} e^{-i P(x-y)} \sum_{l} \frac{2 l+1}{4 \pi} P_{l}(\cos \gamma)(-i) \mathbf{G}^{(E, l)}\left(r, r^{\prime}\right),
$$

where $\gamma$ is the angle between $\vec{r}$ and $\vec{r}^{\prime}$. The variable $E$ in the superscript is the internal energy of the two-body state $\left(E=P^{0}-\vec{P}^{2} / 4 m\right)$. The equation for the function $\mathbf{G}^{(E, l)}\left(r, r^{\prime}\right)$ is then given by

$$
\left(-E-\frac{1}{m r} \frac{d^{2}}{d r^{2}} r-\frac{l(l+1)}{m r^{2}}+\mathbf{V}(r)-i \Gamma \frac{\delta(r)}{2 \pi r^{2}}\right) \mathbf{G}^{(E, l)}\left(r, r^{\prime}\right)=\frac{\delta\left(r-r^{\prime}\right)}{r^{2}} .
$$

The invariant amplitude $\mathcal{M}_{i}^{(S)}(v)$ is written by the $(i, i)$-component of the $S$-wave Green function $\mathbf{G}_{i i}^{(E, 0)}$ as

$$
\mathcal{M}_{i}^{(S)}(v)=\frac{k^{2}}{4 \pi^{4}} \lim _{E \rightarrow k^{2} / m}\left(E-\frac{k^{2}}{m}\right)^{2} \int_{0}^{\infty} r^{2} d r r^{\prime 2} d r^{\prime} j_{0}(k r) j_{0}\left(k r^{\prime}\right) \mathbf{G}_{i i}^{(E, 0)}\left(r, r^{\prime}\right),
$$

where $k=m v / 2$ and $j_{0}(x)$ is the zero-th order of the spherical Bessel function. By using this expression, we obtain the formula for the total $S$-wave annihilation cross section $\sigma_{i}^{(S)}$,

$$
\sigma_{i}^{(S)} v=c_{i} \frac{2 \pi}{k^{2}} \lim _{E \rightarrow k^{2} / m}\left(E-\frac{k^{2}}{m}\right)^{2} \int_{0}^{\infty} r d r r^{\prime} d r^{\prime} \sin (k r) \sin \left(k r^{\prime}\right) \Im\left[\mathbf{G}_{i i}^{(E, 0)}\left(r, r^{\prime}\right)\right] .
$$


In general, the function $\mathbf{G}_{i i}^{(E, 0)}\left(r, r^{\prime}\right)$ can not be solved analytically, therefore we need to solve the Schwinger-Dyson equation (38) numerically to obtain the cross sections.

\section{Solving the Schroedinger equation}

When a function, $\mathbf{g}\left(r, r^{\prime}\right)=r r^{\prime} \mathbf{G}^{(E, 0)}\left(r, r^{\prime}\right)$, is defined, Eq. (38) becomes the standard Schroedinger equation in one dimension,

$$
-\frac{1}{m} \frac{d^{2}}{d r^{2}} \mathbf{g}\left(r, r^{\prime}\right)+\left(\mathbf{V}(r)-i \boldsymbol{\Gamma} \frac{\delta(r)}{2 \pi r^{2}}\right) \mathbf{g}\left(r, r^{\prime}\right)-E \mathbf{g}\left(r, r^{\prime}\right)=\delta\left(r-r^{\prime}\right) .
$$

In the following we expand the solution $\mathbf{g}\left(r, r^{\prime}\right)$ perturbatively by the absorptive part $\Gamma$.

At the leading order, the solution $\mathbf{g}_{0}\left(r, r^{\prime}\right)$ satisfies the equation,

$$
-\frac{1}{m} \frac{d^{2}}{d r^{2}} \mathbf{g}_{0}\left(r, r^{\prime}\right)+\mathbf{V}(r) \mathbf{g}_{0}\left(r, r^{\prime}\right)-E \mathbf{g}_{0}\left(r, r^{\prime}\right)=\delta\left(r-r^{\prime}\right)
$$

The boundary conditions of the equation are determined by the following two requirements; (i) The Green function $\mathbf{G}^{(E, 0)}\left(r, r^{\prime}\right)$ must be finite for any $r$ and $r^{\prime}$. (ii) The Green function has only an out-going wave at $\left|r-r^{\prime}\right| \rightarrow \infty$. Then the solution $\mathbf{g}_{0}\left(r, r^{\prime}\right)$ is obtained as

$$
\mathbf{g}_{0}\left(r, r^{\prime}\right)=m \mathbf{g}_{>}(r) \mathbf{g}_{<}^{T}\left(r^{\prime}\right) \theta\left(r-r^{\prime}\right)+m \mathbf{g}_{<}(r) \mathbf{g}_{>}^{T}\left(r^{\prime}\right) \theta\left(r^{\prime}-r\right)
$$

where $\mathbf{g}_{>(<)}(r)$ is the solution of the homogeneous equation,

$$
-\frac{1}{m} \frac{d^{2}}{d r^{2}} \mathbf{g}_{>(<)}(r)+\mathbf{V}(r) \mathbf{g}_{>(<)}(r)=E \mathbf{g}_{>(<)}(r)
$$

Here, $\mathbf{g}_{>(<)}$is given by a $2 \times 2(3 \times 3)$ matrix in the triplet (doublet) EWIMP case. The solution $\mathbf{g}_{<}(r)$ satisfies the boundary conditions, (i) $\mathbf{g}_{<}(0)=\mathbf{0}$ and (ii) $\mathbf{g}_{<}^{\prime}(0)=\mathbf{1}$, while the conditions for $\mathbf{g}_{>}(r)$ are (i) $\mathbf{g}_{>}(0)=\mathbf{1}$ and (ii) $\mathbf{g}_{>}(r)$ has only an out-going wave at $r \rightarrow \infty$.

At the first order of $\boldsymbol{\Gamma}$, the solution of Eq. (41), $\mathbf{g}_{1}\left(r, r^{\prime}\right)$, is simply given by the leading-order solution $\mathbf{g}_{0}\left(r, r^{\prime}\right)$ as

$$
\mathbf{g}_{1}\left(r, r^{\prime}\right)=-\int d r^{\prime \prime} \mathbf{g}_{0}\left(r, r^{\prime \prime}\right)\left(-i \boldsymbol{\Gamma} \frac{\delta\left(r^{\prime \prime}\right)}{2 \pi r^{\prime \prime 2}}\right) \mathbf{g}_{0}\left(r^{\prime \prime}, r^{\prime}\right)=\frac{i m^{2}}{2 \pi} \mathbf{g}_{>}(r) \boldsymbol{\Gamma} \mathbf{g}_{>}\left(r^{\prime}\right) .
$$

The $S$-wave cross sections in Eq. (40) are proportional to the imaginary part of the Green function $\mathbf{G}_{i i}^{(E, 0)}$. The imaginary part is related to not only the annihilation cross section but also the elastic cross section of the process, $\tilde{\chi}^{0} \tilde{\chi}^{0} \rightarrow \tilde{\chi}^{0} \tilde{\chi}^{0}$ or 
$\tilde{\chi}^{+} \tilde{\chi}^{-} \rightarrow \tilde{\chi}^{+} \tilde{\chi}^{-}$. After extracting the contribution of the annihilation processes from $\Im\left[\mathbf{G}_{i i}^{(E, 0)}\right]$, the total $S$-wave annihilation cross section is obtained as

$$
\begin{aligned}
\sigma_{i}^{(S)} v & =c_{i} \frac{m^{2}}{k^{2}} \lim _{E \rightarrow k^{2} / m}\left(E-\frac{k^{2}}{m}\right)^{2} \sum_{a, b} \Gamma_{a b} \mathcal{A}_{i a} \mathcal{A}_{i b}^{*} \\
\mathcal{A}_{i a} & =\int_{0}^{\infty} d r \sin (k r)\left[\mathbf{g}_{>}(r)\right]_{i a} .
\end{aligned}
$$

It is found that only the asymptotic behavior of the function $\mathbf{g}_{>}(r)$ is relevant in the calculation of the cross sections. The function $\mathbf{g}_{>}(r)$ has only an out-going wave at $r \rightarrow \infty$ as stated above. When the $\mathrm{SU}(2)_{L}$ partners do not appear in the asymptotic state $(E<2 \delta m)$, the function $\mathbf{g}_{>}(r)$ should behave as

$$
\left.\left[\mathbf{g}_{>}(r)\right]_{i j}\right|_{r \rightarrow \infty}=\delta_{i 2} d_{2 j}(E) e^{i \sqrt{m E} r} .
$$

Thus, the total EWIMP pair annihilation cross section is given in a simple form,

$$
\sigma_{2}^{(S)} v=2 \sum_{a, b} \boldsymbol{\Gamma}_{a b} d_{2 a}\left(m v^{2} / 4\right) d_{2 b}^{*}\left(m v^{2} / 4\right)
$$

If the potential term in Eq. (42) is neglected (in other word, the long-distance effects are negligible), the coefficient $d_{2 a}(E)$ is given by $\delta_{2 a}$ and the cross section is simply given by $\sigma_{2}^{(S)} v=2 \boldsymbol{\Gamma}_{22}$, which is consistent with the tree-level cross section as expected. Below we omit the subscript 2 for the EWIMP annihilation cross section for simplicity.

\section{One-loop result in $\tilde{\chi}^{0} \tilde{\chi}^{0} \rightarrow \gamma \gamma$ process}

For demonstration of the validity of Eq. (48), we show that the EWIMP annihilation cross section to two photons agrees with the one-loop cross section when the potential term $\mathbf{V}$ is treated perturbatively.

From Eq. (48), the annihilation cross section to two photons is given by

$$
\left.\sigma^{(S)} v\right|_{\gamma \gamma}=2\left[\boldsymbol{\Gamma}_{\gamma \gamma}\right]_{11}\left|d_{21}\left(m v^{2} / 4\right)\right|^{2}
$$

where the partial absorptive part to two photons $\boldsymbol{\Gamma}_{\gamma \gamma}$ is given in Eq. (28) for the triplet EWIMP case and in Eq. (31) for the doublet EWIMP case, respectively. Here we show only the result in the triplet EWIMP case for simplicity. The coefficient $d_{21}$ is obtained by solving the Schroedinger equation (44). When we expand the solution by the potential term $\mathbf{V}(r),\left[\mathbf{g}_{>}(r)\right]_{21}$ is obtained at leading order as

$$
\left[\mathbf{g}_{>}(r)\right]_{21}=d_{21}(E) e^{i \sqrt{m E} r} \simeq \frac{-m \sqrt{2} \alpha_{2}}{m_{W}+\sqrt{2 m \delta m}} e^{i \sqrt{m E} r}
$$


Here, we take $E<2 \delta m$ and $m \gg m_{W}$. Thus, the annihilation cross section to two photons is given as

$$
\left.\sigma^{(S)} v\right|_{\gamma \gamma} \simeq \frac{4 \pi \alpha^{2} \alpha_{2}^{2}}{m_{W}^{2}}\left(1+\sqrt{\frac{2 m \delta m}{m_{W}^{2}}}\right)^{-2}
$$

This agrees with the result obtained in the full one-loop calculation in a heavy EWIMP mass limit [19], including the correction due to the non-vanishing $\delta m$.

\section{Cross section for $\tilde{\chi}^{0} \tilde{\chi}^{0} \rightarrow \gamma \gamma$ in a limit of $m \rightarrow \infty$}

When the EWIMP mass $m$ is much heavier than the weak gauge boson masses, we can not deal with the potential term perturbatively. The equation (44) can be solved analytically in the limit of $m \rightarrow \infty$, and the qualitative behavior of the cross sections can be discussed. This is because the weak gauge boson masses $m_{W}$ and $m_{Z}$ and the mass difference $\delta m$ can be neglected in this limit. The Schroedinger equation for $\mathbf{g}_{>}(r)$ becomes

$$
-\frac{1}{m} \frac{d^{2}}{d r^{2}} \mathbf{g}_{>}(r)+\frac{1}{r} \mathbf{U g}_{>}(r)=E \mathbf{g}_{>}(r)
$$

where $\mathbf{U}$ is the coefficient matrix for the electroweak potential, and defined by $\mathbf{U}=$ $[r \mathbf{V}(r)]_{r \rightarrow 0}$. Because all forces in the potential become Coulomb-type, the solution of above equation is determined by using the confluent geometric function as

$$
\begin{gathered}
{\left[\mathbf{g}_{>}(r)\right]_{i j}=\sum_{i^{\prime}} \mathcal{O}_{i i^{\prime}} \Lambda_{i^{\prime}}(r) \mathcal{O}_{i^{\prime} j}^{T},} \\
\Lambda_{i}(r)=\Gamma\left(1+\frac{i \lambda_{i}}{2} \sqrt{\frac{m}{E}}\right) W_{-\frac{i \lambda_{i}}{2} \sqrt{\frac{m}{E}}, \frac{1}{2}}(-2 i \sqrt{m E} r) .
\end{gathered}
$$

The function $\Gamma(z)$ is the Euler's Gamma function and $W_{\kappa, \mu}(z)$ is the Whittaker function. The matrix $\mathcal{O}$ is the diagonalization matrix, $\mathcal{O}^{T} \mathbf{U O}=\operatorname{diag} .\left(\lambda_{1}, \cdots\right)$, and $\lambda_{i}$ is the eigenvalue of the matrix $\mathbf{U}$.

The EWIMP annihilation cross section to two photons is then derived as

$$
\begin{aligned}
\sigma^{(S)} v & =\frac{\pi \alpha^{2}}{m^{2}}\left|\sum_{i} \mathcal{O}_{i 2} \Gamma\left(1+\frac{i \lambda_{i}}{2} \sqrt{\frac{m}{E}}\right) \mathcal{O}_{i 1}\right|^{2} \\
& \simeq \begin{cases}2.8 \times 10^{-5} / v m^{2} & \text { (Triplet EWIMP) } \\
3.2 \times 10^{-6} / v m^{2} & \text { (Doublet EWIMP) }\end{cases}
\end{aligned}
$$


in the limit of $m \rightarrow \infty$. Here, we use the approximation $v \ll 1$ to derive the last equation. The cross section behaves as $\sigma v \sim 1 /\left(v m^{2}\right)$, and satisfies the unitarity condition in Eq. (11) as expected.

\section{Annihilation Cross Sections and Zero-Energy Resonances}

When the EWIMP mass is heavy enough so that the the effects of the longdistance force by the electroweak potential cannot be ignored but not heavy enough to take the limit of $m \rightarrow \infty$, we have to solve the Schroedinger equation (44) numerically for the precise annihilation cross sections. In this section we show some numerical results, and discuss the behaviors using a toy model.

\section{Numerical result and zero-energy resonances}

First, we show numerical results for the triplet and doublet EWIMP annihilation cross sections to $\gamma \gamma$ and $W^{+} W^{-}$. In Fig. 15, the cross sections with some fixed mass differences $(\delta m=0.1,1 \mathrm{GeV})$ are shown as functions of $m$. We set to the mass difference $\delta m_{N}$ to be $\delta m_{N}=2 \delta m$ for the doublet EWIMP. In this calculation, the relative velocity of the incident EWIMPs $v / c$ is taken to be $10^{-3}$, which is the typical velocity of dark matter in the galactic halo. We also show the leading-order cross sections in perturbation as dotted lines. When the EWIMP mass $m$ is around 100 $\mathrm{GeV}$, the cross sections to $\gamma \gamma$ and $W^{+} W^{-}$are almost the same as the perturbative ones. However, when $m$ is large enough $(m \gtrsim 0.5 \mathrm{TeV}$ for the triplet EWIMP and

$m \gtrsim 1.5 \mathrm{TeV}$ for the doublet EWIMP), the cross sections are significantly enhanced and have resonance behaviors.

The qualitative behavior of the cross sections, especially around the resonances, may be understood by using a toy model, in which the electroweak potential is approximated by a well potential. Here we discuss the triplet EWIMP case, and the extension to the doublet case is straightforward. Taking $c_{W}=1$ and $\delta m=0$ for simplicity, the electroweak potential is approximated as

$$
\mathbf{V}(r)=\left(\begin{array}{cc}
-b_{1} \alpha_{2} m_{W} & -b_{1} \sqrt{2} \alpha_{2} m_{W} \\
-b_{1} \sqrt{2} \alpha_{2} m_{W} & 0
\end{array}\right) \theta\left(\left(b_{2} m_{W}\right)^{-1}-r\right),
$$

where $b_{1}$ and $b_{2}$ are numerical constants. By comparing the annihilation cross sections to $\gamma \gamma$ in this potential and in the perturbative calculation Eq. (51) for small $m$, we find $b_{1}=8 / 9$ and $b_{2}=2 / 3$. Under this potential, the two-body states of 

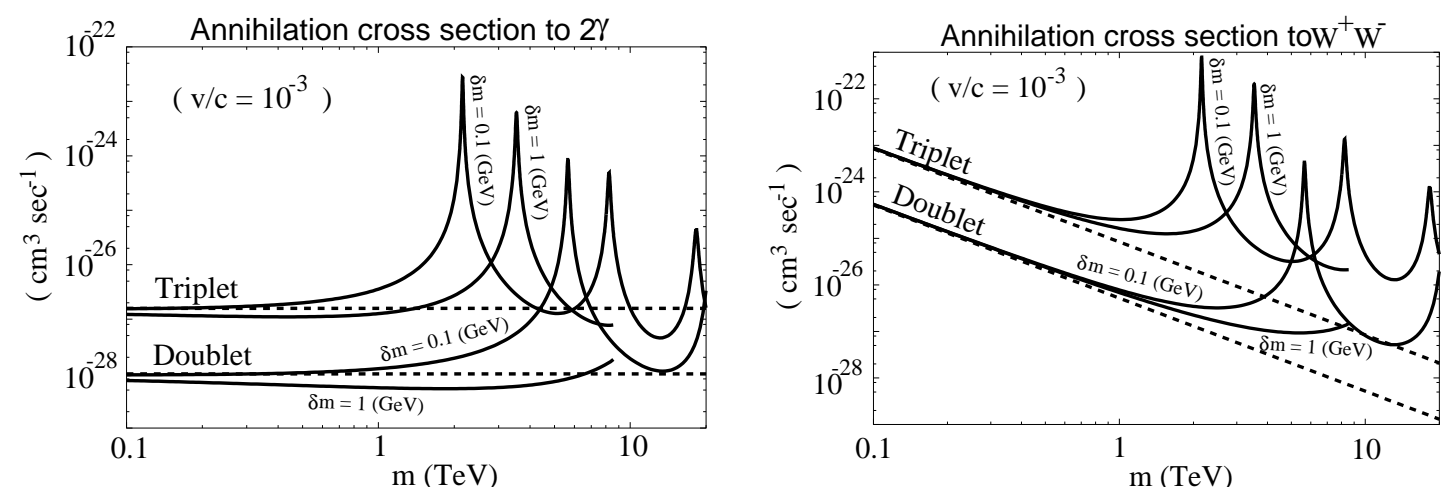

Figure 5: Annihilation cross sections $(\sigma v)$ to $\gamma \gamma$ and $W^{+} W^{-}$when $\delta m=0.1,1 \mathrm{GeV}$ for both the triplet and the doublet EWIMPs. Here, $v / c=10^{-3}$. The leading-order cross sections in perturbation are also shown for $\delta m=0$ (broken lines).

$2 \tilde{\chi}^{0}$ and $\tilde{\chi}^{+} \tilde{\chi}^{-}$have attractive and repulsive states, whose potential energies are $\lambda_{ \pm}=\left(\mathbf{V}_{11} \pm \sqrt{\mathbf{V}_{11}^{2}+4 \mathbf{V}_{12}^{2}}\right) / 2$ with $\mathbf{V}_{i j}(i, j=1,2)$ elements in $\mathbf{V}$. The attractive state is $\cos \theta \phi_{N}-\sin \theta \phi_{C}$ with $\tan ^{2} \theta=-\lambda_{-} / \lambda_{+}$.

By virtue of the approximation, the pair annihilation cross sections for the triplet EWIMP are obtained analytically,

$$
\begin{gathered}
(\sigma v)_{W^{+} W^{-}}=\frac{\pi \alpha_{2}^{2}}{9 m^{2}}\left(\left|d_{21}\right|^{2}+\sqrt{2} \Re\left(d_{21} d_{22}^{*}\right)+2\left|d_{22}\right|^{2}\right), \quad(\sigma v)_{\gamma \gamma}=\frac{2 \pi \alpha^{2}}{9 m^{2}}\left|d_{21}\right|^{2}, \\
d_{21}=\left\{\sqrt{2}\left[\cos \left(p_{c} \sqrt{v^{2} / v_{c}^{2}+2}\right)-i \sqrt{\frac{v^{2}}{v^{2}+2 v_{c}^{2}}} \sin \left(p_{c} \sqrt{v^{2} / v_{c}^{2}+2}\right)\right]^{-1}\right. \\
\left.-\sqrt{2}\left[\cos \left(p_{c} \sqrt{v^{2} / v_{c}^{2}-1}\right)-i \sqrt{\frac{v^{2}}{v^{2}-v_{c}^{2}}} \sin \left(p_{c} \sqrt{v^{2} / v_{c}^{2}-1}\right)\right]^{-1}\right\}, \\
d_{22}=\left\{\begin{array}{r}
\left.\cos \left(p_{c} \sqrt{v^{2} / v_{c}^{2}+2}\right)-i \sqrt{\frac{v^{2}}{v^{2}+2 v_{c}^{2}}} \sin \left(p_{c} \sqrt{v^{2} / v_{c}^{2}+2}\right)\right]^{-1} \\
\left.+2\left[\cos \left(p_{c} \sqrt{v^{2} / v_{c}^{2}-1}\right)-i \sqrt{\frac{v^{2}}{v^{2}-v_{c}^{2}}} \sin \left(p_{c} \sqrt{v^{2} / v_{c}^{2}-1}\right)\right]^{-1}\right\},
\end{array}\right.
\end{gathered}
$$

where $p_{c}$ and $v_{c}$ are defined by $p_{c}=\sqrt{2 \alpha_{2} m / m_{W}}$ and $v_{c}=\sqrt{32 \alpha_{2} m_{W} / 9 m}$, respectively.

If the kinetic energy of the EWIMP pair is much larger than the potential energy $\left(v \gg v_{c}\right)$ or the electroweak potential is point-like $\left(p_{c} \ll 1\right)$, the cross sections 
coincide with the results in leading-order calculation in perturbation as expected. However, when $v$ is much smaller than $v_{c}$, such as in the case of dark matter in the current universe, the $d_{21}$ and $d_{22}$ become

$$
\begin{aligned}
& d_{21} \simeq \sqrt{2}\left[\cos \sqrt{2} p_{c}\right]^{-1}-\sqrt{2}\left[\cosh p_{c}\right]^{-1}, \\
& d_{22} \simeq\left[\cos \sqrt{2} p_{c}\right]^{-1}+2\left[\cosh p_{c}\right]^{-1} .
\end{aligned}
$$

Therefore, when $\sqrt{2} p_{c} \simeq(2 n-1) \pi / 2(n=1,2, \cdots)$, the coefficients $d_{21}$ and $d_{22}$ are enhanced by several orders of magnitude. As the result, the cross sections show the resonance features as in Fig. 5 . These resonances are called zero-energy resonances [30], because the condition $\sqrt{2} p_{c} \simeq(2 n-1) \pi / 2$ is nothing but existence of a bound state with zero binding energy. The bound states consist of mixtures of pairs of the EWIMPs and the charged partners.

In Fig. 5 , the first resonance in small $\delta m$ (that is $\delta m \sim 0.1 \mathrm{GeV}$ ) appears at $m \sim 2 \mathrm{TeV}$. On the other hand, the well potential model predicts the first resonance at $m \sim 1.8 \mathrm{TeV}$. Thus, the model describes the behavior around the first resonance well. Also, notice that the cross section to two photons, which is induced by the one-loop diagrams in perturbation, is suppressed only by $\alpha^{2} / \alpha_{2}^{2}$ compared with that to $W^{+} W^{-}$for $p_{c} \gtrsim 1$. This behavior is also seen in Fig. 囵,

On the resonance, the coefficients $d_{21}$ and $d_{22}$ in Eq. (57) behave as

$$
d_{21} \simeq i \frac{v_{c}}{v}, \quad d_{22} \simeq i \frac{\sqrt{2} v_{c}}{v},
$$

for the small relative velocity $v \ll v_{c}$. Thus the cross sections $\sigma v$ are proportional to $v^{-2}$. However, this is not a signature for breakdown of the unitarity condition in Eq. (11). We find from study in the one-flavor system under the well potential $V$ that $\sigma v$ would be saturated by the finite width for the two-body system (that is, the absorptive part) $\Gamma$ when $v \ll m V \Gamma$, and the unitarity is not broken.

So far we have ignored the mass difference $\delta m$ in Eq. (55). When $\delta m$ is not negligible compared with $\sim \alpha_{2} m_{W}$, the potential energy for the attractive state is reduced as $\left|\lambda_{-}\right| \simeq 16 \alpha_{2} m_{W} / 9-4 \delta m / 3$ for $\delta m \lesssim \alpha_{2} m_{W}$ and $\left|\lambda_{-}\right| \simeq 128\left(\alpha_{2} m_{W}\right)^{2} /(81 \delta m)$ for $\delta m \gtrsim \alpha_{2} m_{W}$, and the attractive force becomes weaker. Also, the component of the EWIMP pair in the attractive state becomes smaller. Then, the zero-energy resonances move to heavier $m$ for larger $\delta m$, and the EWIMP annihilation cross sections around the resonances become smaller, as in Fig. 15. This effect is more significant in the doublet EWIMP case than in the triplet one, since the potential energy by the weak gauge boson exchanges is smaller. 
Now we have discussed importance of the zero-energy resonances for the EWIMP annihilation cross sections. One might consider that the thermal relic EWIMP abundance is also modified due to the zero-energy resonances. The answer to the question is "No". The typical relative velocity is given by $v \sim 1 / 3$ at the freeze out temperature $T \sim m / 20$. Note that the critical velocity $v_{c}$ for the triplet EWIMP is given by $v_{c} \sim 2 \alpha_{2} \sim 0.07$ at the first zero-energy resonance where $\sqrt{2} p_{c}=\pi / 2$. The critical velocity for the doublet EWIMP is smaller than that of the triplet one. Thus the relative velocity at the freeze-out temperature is not small enough to affect the thermal relic abundance.

One might have another question relevant to the annihilation in the universe at very low temperature. Since the cross sections are proportional to $v^{-2}$ on the zero-energy resonance, the EWIMP annihilation rate (the annihilation cross section multiplied by the EWIMP number density $n_{\mathrm{EW}}$ ) becomes much larger after the freezing-out phenomenon stated above. If the annihilation rate becomes larger than the Hubble constant $H$ at low temperature, the EWIMP begins to annihilate again (re-coupling). The usual calculation of dark matter abundance might be changed.

For studying the possibility, we use the previous toy model for the triplet EWIMP. The thermal-averaged annihilation cross section $\langle\sigma v\rangle$ on the first zero-energy resonance at temperature $T$ is derived as

$$
\langle\sigma v\rangle=q\left(\frac{1 \mathrm{GeV}}{T}\right), \quad\left(\text { in the unit of } \mathrm{GeV}^{-2}\right)
$$

where $q \sim 7 \times 10^{-9}$. It is found that the condition of the 're-coupling', $\langle\sigma v\rangle n_{\mathrm{EW}}>H$, attributes to the inequality,

$$
\frac{\rho_{\mathrm{EW}}}{s} \geq 7 \times 10^{-8}[\mathrm{GeV}]
$$

where $\rho_{\mathrm{EW}}\left(\equiv m n_{\mathrm{EW}}\right)$ is the EWIMP mass density and $s$ is the entropy of the universe. The lower bound is larger than the cosmological observation for dark matter $\left(\rho / s \simeq 7 \times 10^{-9} \mathrm{GeV}\right)$. Thus the re-coupling does not occur in our universe.

\section{Fitting functions}

The numerical calculation of the EWIMP annihilation cross sections takes huge CPU power. It is thus convenient to derive the fitting functions which reproduce the numerical results with enough precision. We performed two parameter fitting of the annihilation cross sections for the doublet and triplet EWIMPs, the EWIMP mass 
$m$ and the mass difference between the EWIMP and its charged partner $\delta m$. The velocity dependence of the cross sections is weak except for very narrow regions in the vicinity of the resonances and ignored. For the doublet EWIMP, the cross sections further depend on the mass difference between the EWIMP and its neutral partner $\delta m_{N}$. We fix $\delta m_{N}=2 \delta m$ in the derivation of the fitting functions for simplicity. This relation is valid in the MSSM when $\tan \beta$ is large.

We use the fitting function,

$$
\left.\sigma v(m, \delta m)\right|_{\mathrm{fit}}=10^{-28}\left[\mathrm{~cm}^{3} \mathrm{~s}^{-1}\right] \times\left[\sum_{i, j=0}^{6} a_{i j}\left(\frac{m}{1 \mathrm{TeV}}\right)^{i}\left(\frac{\delta m}{1 \mathrm{GeV}}\right)^{j}\right]^{-2},
$$

in the range $0.2 \mathrm{TeV} \leq m \leq 10 \mathrm{TeV}$ and $0.1 \mathrm{GeV} \leq \delta m \leq 1 \mathrm{GeV}$. By performing the least-square method between the fitting function and the numerical integration, the coefficients $a_{i j}$ in each process are obtained. The numerical values of the coefficients $a_{i j}$ for the annihilation cross sections to $\gamma \gamma$ and $W^{+} W^{-}$in the triplet EWIMP case and $\gamma \gamma, W^{+} W^{-}$and $Z^{0} Z^{0}$ in the doublet EWIMP case are given in Table 1 . Other annihilation cross sections are evaluated from the cross sections to two photons as

$$
\begin{aligned}
\sigma v\left(\tilde{\chi}^{0} \tilde{\chi}^{0} \rightarrow \gamma Z^{0}\right) & =\sigma v\left(\tilde{\chi}^{0} \tilde{\chi}^{0} \rightarrow \gamma \gamma\right) \times \frac{2 \alpha_{2} c_{W}^{2}}{\alpha}, \\
\sigma v\left(\tilde{\chi}^{0} \tilde{\chi}^{0} \rightarrow Z^{0} Z^{0}\right) & =\sigma v\left(\tilde{\chi}^{0} \tilde{\chi}^{0} \rightarrow \gamma \gamma\right) \times \frac{\alpha_{2}^{2} c_{W}^{4}}{\alpha^{2}},
\end{aligned}
$$

in the triplet EWIMP case, and

$$
\sigma v\left(\tilde{\chi}^{0} \tilde{\chi}^{0} \rightarrow \gamma Z^{0}\right)=\sigma v\left(\tilde{\chi}^{0} \tilde{\chi}^{0} \rightarrow \gamma \gamma\right) \times \frac{\alpha_{2}\left(1-2 s_{W}^{2}\right)^{2}}{2 \alpha c_{W}^{2}}
$$

in the doublet EWIMP case. This is because these processes are induced via the transition of the EWIMP pair to the charged partner pair.

\section{Annihilation cross sections in the MSSM parameters}

By using above fitting functions, we scan the annihilation cross sections on the MSSM parameters. The input parameters are the Bino mass $M_{1}$, the Wino mass $M_{2}$, the Higgsino mass $\mu$, and $\tan \beta$. We assume that the other supersymmetric scalar particles, sleptons and squarks, are heavy enough, and neglect the contributions to the cross sections. Furthermore we consider two situations, in which relations between $M_{1}$ and $M_{2}$ are different. The first one is the GUT relation $M_{1}=0.5 M_{2}$, which is frequently assumed in the minimal supergravity scenario. Another one is 
(Triplet EWIMP, to $\gamma \gamma$ )

\begin{tabular}{|c||r|r|r|r|r|r|r|}
\hline$a_{i j}$ & $\mathrm{j}=0$ & \multicolumn{1}{c|}{$\mathrm{j}=1$} & $\mathrm{j}=2$ & $\mathrm{j}=3$ & $\mathrm{j}=4$ & \multicolumn{1}{c|}{$\mathrm{j}=5$} & $\mathrm{j}=6$ \\
\hline $\mathrm{i}=0$ & $2.56521 \mathrm{E}-1$ & $2.88649 \mathrm{E}-2$ & $1.24874 \mathrm{E}-1$ & $-4.97529 \mathrm{E}-1$ & $8.48760 \mathrm{E}-1$ & $-6.97638 \mathrm{E}-1$ & $2.23588 \mathrm{E}-1$ \\
\hline $\mathrm{i}=1$ & $-1.10191 \mathrm{E}-1$ & $8.27605 \mathrm{E}-1$ & $-3.13417 \mathrm{E}+0$ & $7.52692 \mathrm{E}+0$ & $-1.02429 \mathrm{E}+1$ & $7.27770 \mathrm{E}+0$ & $-2.09385 \mathrm{E}+0$ \\
\hline $\mathrm{i}=2$ & $-1.59340 \mathrm{E}-2$ & $-4.83605 \mathrm{E}-1$ & $2.29288 \mathrm{E}+0$ & $-5.90629 \mathrm{E}+0$ & $8.31712 \mathrm{E}+0$ & $-6.03157 \mathrm{E}+0$ & $1.75985 \mathrm{E}+0$ \\
\hline $\mathrm{i}=3$ & $-8.78312 \mathrm{E}-3$ & $2.29927 \mathrm{E}-1$ & $-1.11637 \mathrm{E}+0$ & $2.82055 \mathrm{E}+0$ & $-3.86927 \mathrm{E}+0$ & $2.73075 \mathrm{E}+0$ & $-7.76046 \mathrm{E}-1$ \\
\hline $\mathrm{i}=4$ & $4.66594 \mathrm{E}-3$ & $-5.84155 \mathrm{E}-2$ & $2.72064 \mathrm{E}-1$ & $-6.65170 \mathrm{E}-1$ & $8.86046 \mathrm{E}-1$ & $-6.08312 \mathrm{E}-1$ & $1.68406 \mathrm{E}-1$ \\
\hline $\mathrm{i}=5$ & $-5.44363 \mathrm{E}-4$ & $6.00290 \mathrm{E}-3$ & $-2.73828 \mathrm{E}-2$ & $6.56610 \mathrm{E}-2$ & $-8.58639 \mathrm{E}-2$ & $5.79081 \mathrm{E}-2$ & $-1.57571 \mathrm{E}-2$ \\
\hline $\mathrm{i}=6$ & $1.95407 \mathrm{E}-5$ & $-2.10046 \mathrm{E}-4$ & $9.48764 \mathrm{E}-4$ & $-2.24620 \mathrm{E}-3$ & $2.89895 \mathrm{E}-3$ & $-1.92989 \mathrm{E}-3$ & $5.18540 \mathrm{E}-4$ \\
\hline
\end{tabular}

(Triplet EWIMP, to $W^{+} W^{-}$)

\begin{tabular}{|c||r|r|r|r|r|r|r|}
\hline$a_{i j}$ & $\mathrm{j}=0$ & \multicolumn{1}{c|}{$\mathrm{j}=1$} & $\mathrm{j}=2$ & $\mathrm{j}=3$ & $\mathrm{j}=4$ & $\mathrm{j}=5$ & $\mathrm{j}=6$ \\
\hline $\mathrm{i}=0$ & $-3.36985 \mathrm{E}-3$ & $1.23215 \mathrm{E}-2$ & $-4.08164 \mathrm{E}-2$ & $5.96407 \mathrm{E}-2$ & $-2.63576 \mathrm{E}-2$ & $-1.42661 \mathrm{E}-2$ & $1.13721 \mathrm{E}-2$ \\
\hline $\mathrm{i}=1$ & $5.61768 \mathrm{E}-2$ & $-6.02547 \mathrm{E}-2$ & $1.31888 \mathrm{E}-1$ & $-3.54816 \mathrm{E}-2$ & $-2.97169 \mathrm{E}-1$ & $4.06116 \mathrm{E}-1$ & $-1.57962 \mathrm{E}-1$ \\
\hline $\mathrm{i}=2$ & $-4.18499 \mathrm{E}-2$ & $8.09353 \mathrm{E}-2$ & $-1.05570 \mathrm{E}-1$ & $-1.60918 \mathrm{E}-1$ & $6.66742 \mathrm{E}-1$ & $-7.10708 \mathrm{E}-1$ & $2.52266 \mathrm{E}-1$ \\
\hline $\mathrm{i}=3$ & $6.54865 \mathrm{E}-3$ & $-4.19556 \mathrm{E}-4$ & $-8.61235 \mathrm{E}-2$ & $3.59611 \mathrm{E}-1$ & $-6.42137 \mathrm{E}-1$ & $5.31727 \mathrm{E}-1$ & $-1.66897 \mathrm{E}-1$ \\
\hline $\mathrm{i}=4$ & $1.43330 \mathrm{E}-4$ & $-6.78893 \mathrm{E}-3$ & $4.15698 \mathrm{E}-2$ & $-1.20926 \mathrm{E}-1$ & $1.83077 \mathrm{E}-1$ & $-1.37981 \mathrm{E}-1$ & $4.08085 \mathrm{E}-2$ \\
\hline $\mathrm{i}=5$ & $-8.33119 \mathrm{E}-5$ & $1.03757 \mathrm{E}-3$ & $-5.22051 \mathrm{E}-3$ & $1.36654 \mathrm{E}-2$ & $-1.93028 \mathrm{E}-2$ & $1.38678 \mathrm{E}-2$ & $-3.96380 \mathrm{E}-3$ \\
\hline $\mathrm{i}=6$ & $4.08625 \mathrm{E}-6$ & $-4.38210 \mathrm{E}-5$ & $2.04733 \mathrm{E}-4$ & $-5.07069 \mathrm{E}-4$ & $6.86353 \mathrm{E}-4$ & $-4.77300 \mathrm{E}-4$ & $1.33068 \mathrm{E}-4$ \\
\hline
\end{tabular}

(Doublet EWIMP, to $\gamma \gamma$ )

\begin{tabular}{|c||r|r|r|r|r|r|r|}
\hline$a_{i j}$ & \multicolumn{1}{|c|}{$\mathrm{j}=0$} & $\mathrm{j}=1$ & $\mathrm{j}=2$ & \multicolumn{1}{c|}{$\mathrm{j}=3$} & \multicolumn{1}{c|}{$\mathrm{j}=4$} & $\mathrm{j}=5$ & $\mathrm{j}=6$ \\
\hline $\mathrm{i}=0$ & $1.04998 \mathrm{E}+0$ & $-1.92220 \mathrm{E}-1$ & $2.60536 \mathrm{E}+0$ & $-8.25766 \mathrm{E}+0$ & $1.24256 \mathrm{E}+1$ & $-9.11921 \mathrm{E}+0$ & $2.61707 \mathrm{E}+0$ \\
\hline $\mathrm{i}=1$ & $-4.17041 \mathrm{E}-1$ & $4.24814 \mathrm{E}+0$ & $-1.96145 \mathrm{E}+1$ & $5.23145 \mathrm{E}+1$ & $-7.39832 \mathrm{E}+1$ & $5.28533 \mathrm{E}+1$ & $-1.49773 \mathrm{E}+1$ \\
\hline $\mathrm{i}=2$ & $1.03008 \mathrm{E}-1$ & $-1.93097 \mathrm{E}+0$ & $1.05626 \mathrm{E}+1$ & $-2.97310 \mathrm{E}+1$ & $4.29137 \mathrm{E}+1$ & $-3.10093 \mathrm{E}+1$ & $8.82103 \mathrm{E}+0$ \\
\hline $\mathrm{i}=3$ & $-2.42293 \mathrm{E}-2$ & $4.15616 \mathrm{E}-1$ & $-2.28085 \mathrm{E}+0$ & $6.20020 \mathrm{E}+0$ & $-8.49779 \mathrm{E}+0$ & $5.80186 \mathrm{E}+0$ & $-1.52947 \mathrm{E}+0$ \\
\hline $\mathrm{i}=4$ & $2.24803 \mathrm{E}-3$ & $-3.43865 \mathrm{E}-2$ & $1.66100 \mathrm{E}-1$ & $-3.65744 \mathrm{E}-1$ & $3.37953 \mathrm{E}-1$ & $-9.30267 \mathrm{E}-2$ & $-2.82121 \mathrm{E}-2$ \\
\hline $\mathrm{i}=5$ & $-6.51687 \mathrm{E}-5$ & $6.85671 \mathrm{E}-4$ & $-2.51709 \mathrm{E}-4$ & $-1.22816 \mathrm{E}-2$ & $4.22829 \mathrm{E}-2$ & $-5.14471 \mathrm{E}-2$ & $2.24206 \mathrm{E}-2$ \\
\hline $\mathrm{i}=6$ & $-1.47604 \mathrm{E}-7$ & $1.74390 \mathrm{E}-5$ & $-2.40349 \mathrm{E}-4$ & $1.19010 \mathrm{E}-3$ & $-2.71981 \mathrm{E}-3$ & $2.87046 \mathrm{E}-3$ & $-1.16460 \mathrm{E}-3$ \\
\hline
\end{tabular}

(Doublet EWIMP, to $W^{+} W^{-}$)

\begin{tabular}{|c||r|r|r|r|r|r|r|}
\hline$a_{i j}$ & $\mathrm{j}=0$ & $\mathrm{j}=1$ & $\mathrm{j}=2$ & $\mathrm{j}=3$ & $\mathrm{j}=4$ & $\mathrm{j}=5$ & $\mathrm{j}=6$ \\
\hline $\mathrm{i}=0$ & $-6.51180 \mathrm{E}-3$ & $2.79481 \mathrm{E}-2$ & $-5.26301 \mathrm{E}-2$ & $4.49224 \mathrm{E}-2$ & $9.13789 \mathrm{E}-3$ & $-4.38570 \mathrm{E}-2$ & $2.11067 \mathrm{E}-2$ \\
\hline $\mathrm{i}=1$ & $1.71330 \mathrm{E}-1$ & $-1.78724 \mathrm{E}-1$ & $5.13363 \mathrm{E}-1$ & $-8.98539 \mathrm{E}-1$ & $8.56009 \mathrm{E}-1$ & $-3.75230 \mathrm{E}-1$ & $4.87411 \mathrm{E}-2$ \\
\hline $\mathrm{i}=2$ & $-5.42160 \mathrm{E}-2$ & $1.85541 \mathrm{E}-1$ & $-5.40450 \mathrm{E}-1$ & $9.57081 \mathrm{E}-1$ & $-8.89508 \mathrm{E}-1$ & $3.57243 \mathrm{E}-1$ & $-3.19227 \mathrm{E}-2$ \\
\hline $\mathrm{i}=3$ & $2.44545 \mathrm{E}-3$ & $-9.05849 \mathrm{E}-4$ & $-3.64007 \mathrm{E}-2$ & $1.57625 \mathrm{E}-1$ & $-3.13715 \mathrm{E}-1$ & $2.94701 \mathrm{E}-1$ & $-1.03643 \mathrm{E}-1$ \\
\hline $\mathrm{i}=4$ & $2.49469 \mathrm{E}-4$ & $-3.59210 \mathrm{E}-3$ & $2.13727 \mathrm{E}-2$ & $-6.52043 \mathrm{E}-2$ & $1.08466 \mathrm{E}-1$ & $-9.07532 \mathrm{E}-2$ & $2.95480 \mathrm{E}-2$ \\
\hline $\mathrm{i}=5$ & $-2.49263 \mathrm{E}-5$ & $3.33127 \mathrm{E}-4$ & $-2.02985 \mathrm{E}-3$ & $6.39148 \mathrm{E}-3$ & $-1.07614 \mathrm{E}-2$ & $9.00477 \mathrm{E}-3$ & $-2.92073 \mathrm{E}-3$ \\
\hline $\mathrm{i}=6$ & $6.51335 \mathrm{E}-7$ & $-9.63636 \mathrm{E}-6$ & $6.24283 \mathrm{E}-5$ & $-2.04190 \mathrm{E}-4$ & $3.50050 \mathrm{E}-4$ & $-2.95151 \mathrm{E}-4$ & $9.60757 \mathrm{E}-5$ \\
\hline
\end{tabular}

(Doublet EWIMP, to $Z^{0} Z^{0}$ )

\begin{tabular}{|c|c|c|c|c|c|c|c|}
\hline$a_{i j}$ & $\mathrm{j}=0$ & $\mathrm{j}=1$ & $\mathrm{j}=2$ & $\mathrm{j}=3$ & $\mathrm{j}=4$ & $\mathrm{j}=5$ & $\mathrm{j}=6$ \\
\hline$i=0$ & $-4.38874 \mathrm{E}-5$ & $-9.72321 \mathrm{E}-3$ & $7.53513 \mathrm{E}-2$ & $-2.21045 \mathrm{E}-1$ & $3.21256 \mathrm{E}-1$ & $-2.32073 \mathrm{E}-1$ & $6.64397 \mathrm{E}-2$ \\
\hline$i=1$ & $1.44964 \mathrm{E}-1$ & $8.69220 \mathrm{E}-2$ & $-5.06389 \mathrm{E}-1$ & $1.34705 \mathrm{E}+0$ & $-1.86944 \mathrm{E}+0$ & $1.31757 \mathrm{E}+0$ & $-3.72003 \mathrm{E}-1$ \\
\hline$i=2$ & $-6.16430 \mathrm{E}-3$ & $-1.64703 \mathrm{E}-1$ & $8.62852 \mathrm{E}-1$ & $-2.17291 \mathrm{E}+0$ & $2.92730 \mathrm{E}+0$ & $-2.02460 \mathrm{E}+0$ & $5.63888 \mathrm{E}-1$ \\
\hline $\mathrm{i}=3$ & $-1.36278 \mathrm{E}-2$ & $1.29107 \mathrm{E}-1$ & $-5.70725 \mathrm{E}-1$ & $1.35708 \mathrm{E}+0$ & $-1.77804 \mathrm{E}+0$ & $1.20897 \mathrm{E}+0$ & $-3.32609 \mathrm{E}-1$ \\
\hline $\mathrm{i}=4$ & $2.05191 \mathrm{E}-3$ & $-1.94524 \mathrm{E}-2$ & $8.76460 \mathrm{E}-2$ & $-2.13319 \mathrm{E}-1$ & $2.86459 \mathrm{E}-1$ & $-1.99525 \mathrm{E}-1$ & $5.61327 \mathrm{E}-2$ \\
\hline $\mathrm{i}=5$ & $-1.06900 \mathrm{E}-4$ & $1.07990 \mathrm{E}-3$ & $-5.08739 \mathrm{E}-3$ & $1.28877 \mathrm{E}-2$ & $-1.79673 \mathrm{E}-2$ & $1.29591 \mathrm{E}-2$ & $-3.76293 \mathrm{E}-3$ \\
\hline$i=6$ & $1.93736 \mathrm{E}-6$ & $-2.09370 \mathrm{E}-5$ & $1.03637 \mathrm{E}-4$ & $-2.74183 \mathrm{E}-4$ & $3.97811 \mathrm{E}-4$ & $-2.97743 \mathrm{E}-4$ & $8.93856 \mathrm{E}-5$ \\
\hline
\end{tabular}

Table 1: Coefficients of the fitting function Eq. (61) for the EWIMP annihilation cross sections to $\gamma \gamma$ and $W^{+} W^{-}$in the triplet case, and to $\gamma \gamma, W^{+} W^{-}$and $Z^{0} Z^{0}$ in the doublet

case. 
$M_{1}=3 M_{2}$, which is predicted in the anomaly mediated supersymmetry breaking scenario. The lightest neutralino mass $m$ and the mass difference between the neutralino and the chargino $\delta m$ in these two cases are given in Fig. [1 and Fig. 2 in Sec. II.

The non-perturbative effects due to the existence of the resonances are important for the calculation of the annihilation cross sections when the neutralino is Wino- or Higgsino-like and heavy enough. On the other hand, the leading-order calculation is precise enough in other regions of the parameters. We thus match the fitting functions derived in the previous section to the leading-order cross sections in perturbation at $m=250 \mathrm{GeV}$ and $\delta m=1 \mathrm{GeV}$ with $\left|Z_{12}\right|^{2} \geq 0.9$ for the Wino-like and $\left|Z_{13}\right|^{2}+\left|Z_{14}\right|^{2} \geq 0.9$ for the Higgsino-like neutralino. Here, $Z_{i j}$ is defined in Eq. (4).

The results are shown in Fig. 6] and Fig. [7 which are contour plots of the cross sections to $W^{+} W^{-}$and $\gamma \gamma$. In Fig. 6, the relation $M_{1}=0.5 M_{2}$ is imposed. The regions $|\mu| \lesssim 0.5 M_{2}$ correspond to the Higgsino-like regions. As in Fig. 11 the mass difference between the neutralino and the chargino is larger than $1 \mathrm{GeV}$ in most of the parameter space. As the result, the first resonance appears at $|\mu| \sim 10 \mathrm{TeV}$, which is out of the range of these figures. In the regions with $0.5 M_{2} \lesssim|\mu|$ the LSP is the Bino-like neutralino.

The relation $M_{1}=3 M_{2}$ is imposed in Fig. 7]. In addition to the Higgsino-like regions, which appears in the same place as ones in Fig. 6, the Wino-like regions also appear in the regions $M_{2} \lesssim|\mu|$. The first zero-energy resonance of the Wino-like neutralino is shown up at $M_{2} \sim 2 \mathrm{TeV}$.

We also show the ratios of the annihilation cross sections including the effects of the resonances and the leading-order ones in perturbation in Fig. 8 and Fig. 9 , All parameters for depicting these figures are the same as the those for Fig. [6 and Fig. 17. Huge enhancements are found in the vicinities of the zero-energy resonances. Furthermore, the cross sections are enhanced by factors even for $m \gtrsim 500 \mathrm{GeV}$ when the lightest neutralino is Wino-like. Thus, the non-perturbative effect is important to obtain the precise annihilation cross sections. The ratios in the Higgsino-like regions in Fig. 9 are larger than that in Fig. 8. This is because the mass difference $\delta m$ is smaller in Fig. 9 than in Fig. 8. The non-perturbative effects should also be included for the Higgsino-like neutralino with $m \gtrsim 500 \mathrm{GeV}$ when the accurate cross sections are required. 

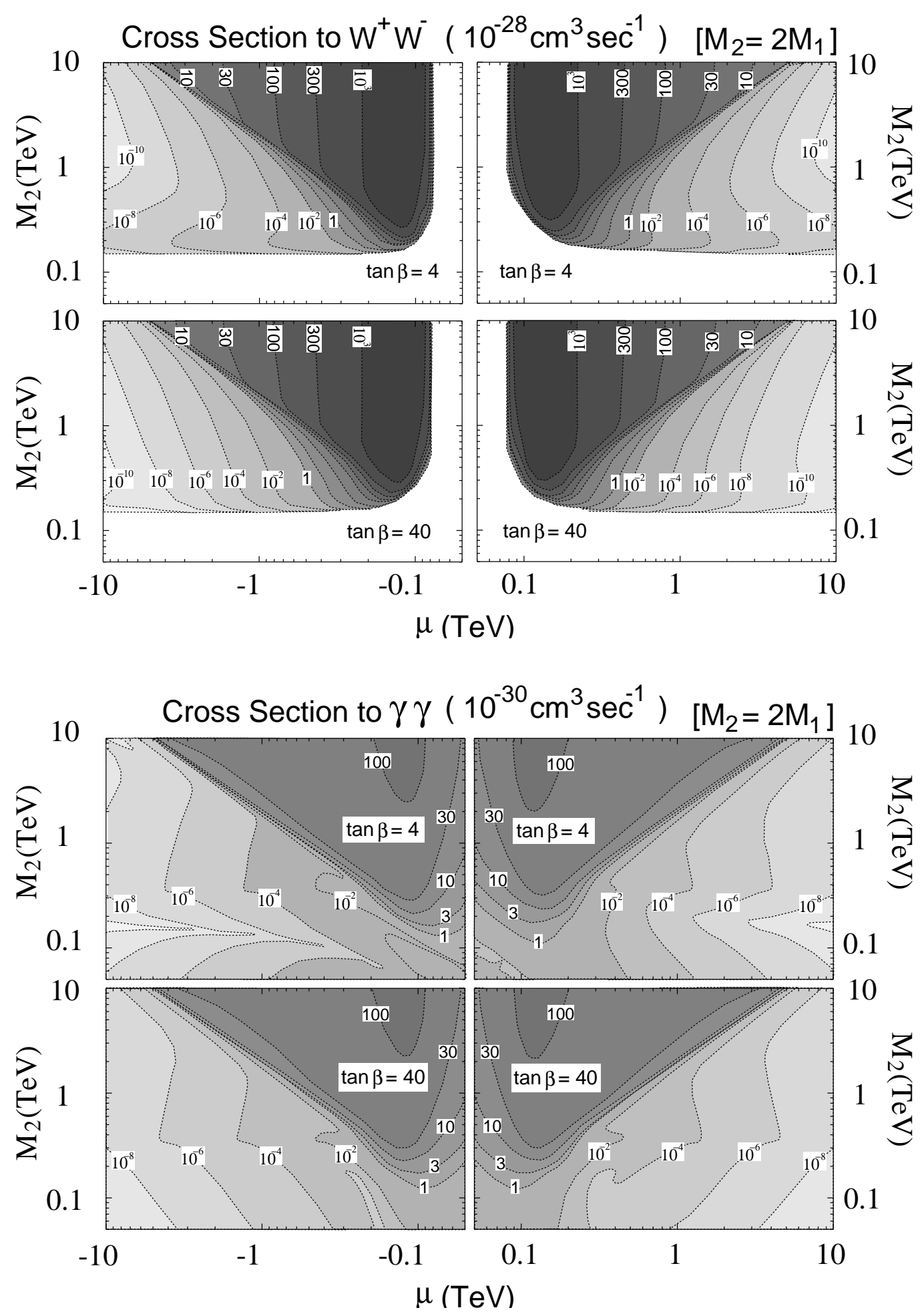

Figure 6: Contour maps of the neutralino annihilation cross sections $(\sigma v)$ to $W^{+} W^{-}$ (four top figures) and $\gamma \gamma$ (four bottom figures) in $\left(M_{2}, \mu\right)$ planes with $\tan \beta=4,40$ in the MSSM. $M_{1}=0.5 M_{2}$ is assumed. 

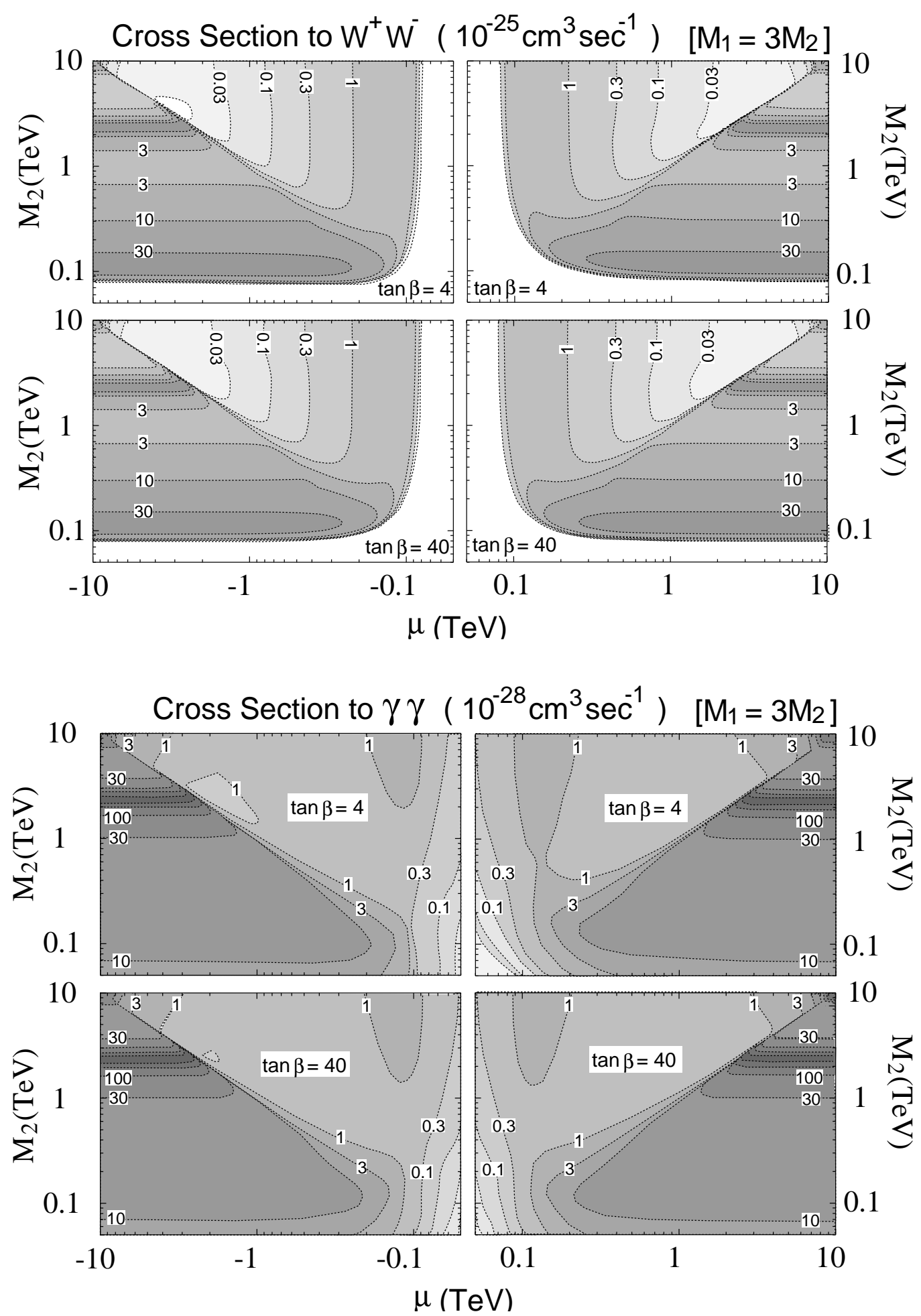

Figure 7: Contour maps of the neutralino annihilation cross sections $(\sigma v)$ to $W^{+} W^{-}$ (four top figures) and $\gamma \gamma$ (four bottom figures) in $\left(M_{2}, \mu\right)$ planes with $\tan \beta=4,40$ in the MSSM. $M_{1}=3 M_{2}$ is assumed. 

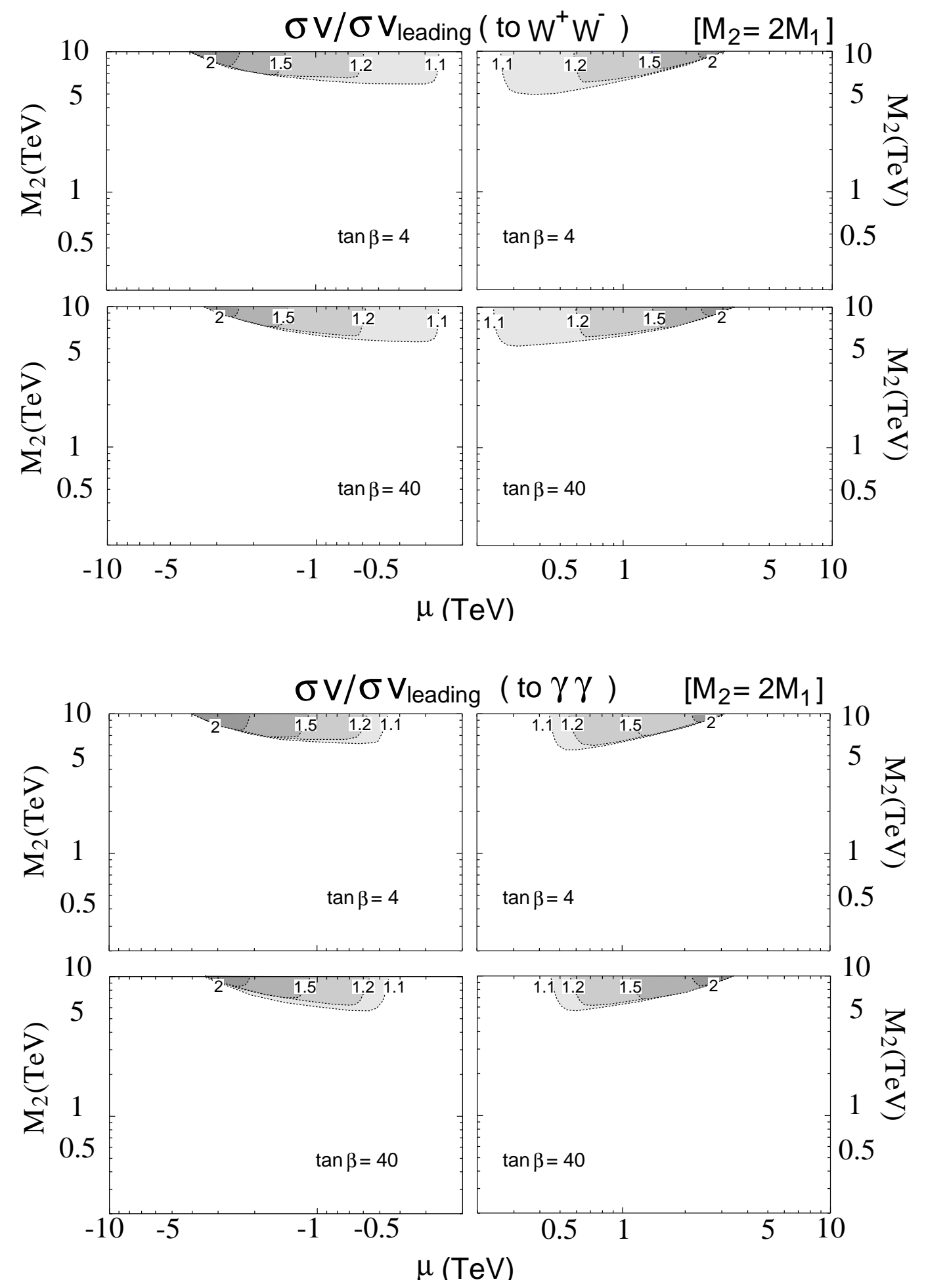

Figure 8: Ratios of the cross sections including the non-perturbative effects and the leading-order ones in perturbation, $\sigma / \sigma_{\text {leading }}$, in the MSSM. Figures are shown as contour maps in $\left(M_{2}, \mu\right)$ planes. All parameters needed for depicting the lines are the same as those in Fig. 6. 

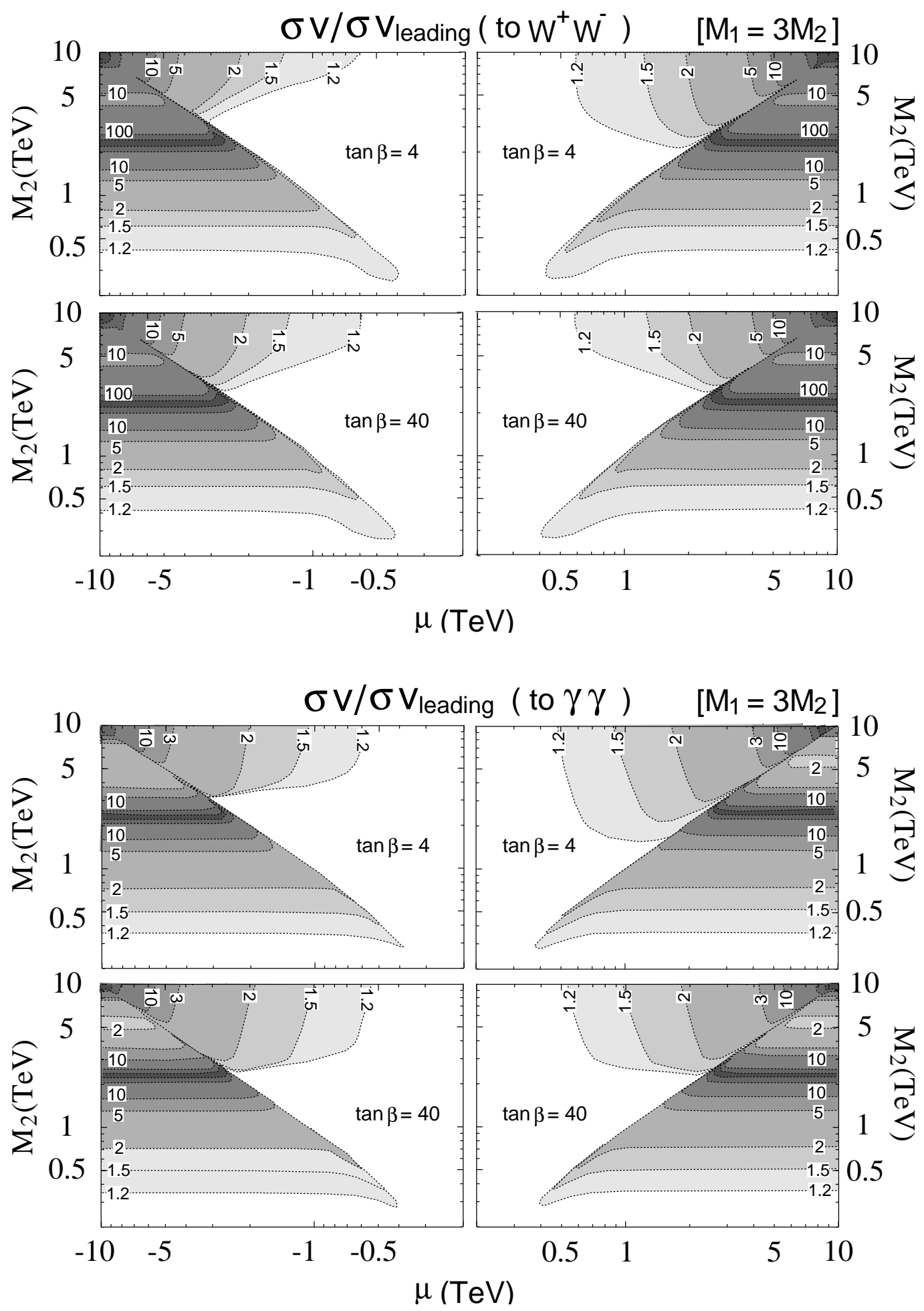

Figure 9: Ratios of the cross sections including the non-perturbative effects and the leading-order ones in perturbation, $\sigma / \sigma_{\text {leading }}$, in the MSSM. Figures are shown as contour maps in $\left(M_{2}, \mu\right)$ planes. All parameters needed for depicting the lines are the same as those in Fig. 7 


\section{Gamma Ray Flux from the Galactic Center}

The enhancement of the dark matter annihilation cross sections has significant implication for indirect searches for dark matter using cosmic rays. In this section, we discuss the searches for gamma rays resulting from the dark matter annihilation in the galactic center and the future prospects.

\section{Flux formula}

The spectrum of gamma rays from the dark matter annihilation consists of two components. One is the line gamma rays and the other is the continuum gamma rays. The line gamma rays are produced by the radiative processes such as the dark matter annihilations to $\gamma \gamma$ and $Z \gamma$ [19]. Since the dark matter is non-relativistic in the galactic halo, the resulting spectrum is monochromatic. The signal is robust for the dark matter search, because the diffused gamma-ray background from the astrophysical sources has a continuous energy spectrum.

The continuum gamma ray signal come from jets in the dark matter annihilation. For example, the dark matter annihilates to $W$ bosons, the $W$ bosons fragment into $\pi$ mesons and finally $\pi^{0}$ mesons decay to $\gamma \gamma$. The energy spectrum thus becomes continuous. The observation of the continuum gamma rays may also constrain the properties of dark matter if the astrophysical background is understood well.

The gamma ray flux from the dark matter annihilation, $\mathcal{F}_{\gamma}(E)$, is given by

$$
\frac{d \mathcal{F}_{\gamma}(\theta, E)}{d \Omega d E}=\frac{1}{4 \pi m^{2}} \sum_{f} \frac{d N_{f}^{(\gamma)}}{d E} \frac{\langle\sigma v\rangle_{f}}{2} \int_{\text {line of sight }} d l(\theta) \rho^{2}(l)
$$

where $\theta$ is the angle between the direction of the galactic center and that of ob-

servation. The function $N_{f}^{(\gamma)}(E)$ is the number of photons with energy $E$ in the fragmentation of the final state $f$, and $\langle\sigma v\rangle$ is the dark matter annihilation cross section averaged with respect to the velocity distribution function. The density $\rho$ in the integrand is the dark matter mass density profile in our galaxy.

After integrating out Eq. (64) by the solid angle with the appropriate angular resolution of the detector, we obtain

$$
\begin{aligned}
\frac{d \mathcal{F}_{\gamma}(E)}{d E} & =9.3 \times 10^{-12}\left[\mathrm{~cm}^{-2} \sec ^{-1} \mathrm{GeV}^{-1}\right] \\
& \times\left(\frac{100 \mathrm{GeV}}{m}\right)^{2} \sum_{f} \frac{d N_{f}^{(\gamma)}}{d E}\left(\frac{\langle\sigma v\rangle_{f}}{10^{-27} \mathrm{~cm}^{3} \mathrm{sec}^{-1}}\right) \bar{J} \Delta \Omega .
\end{aligned}
$$



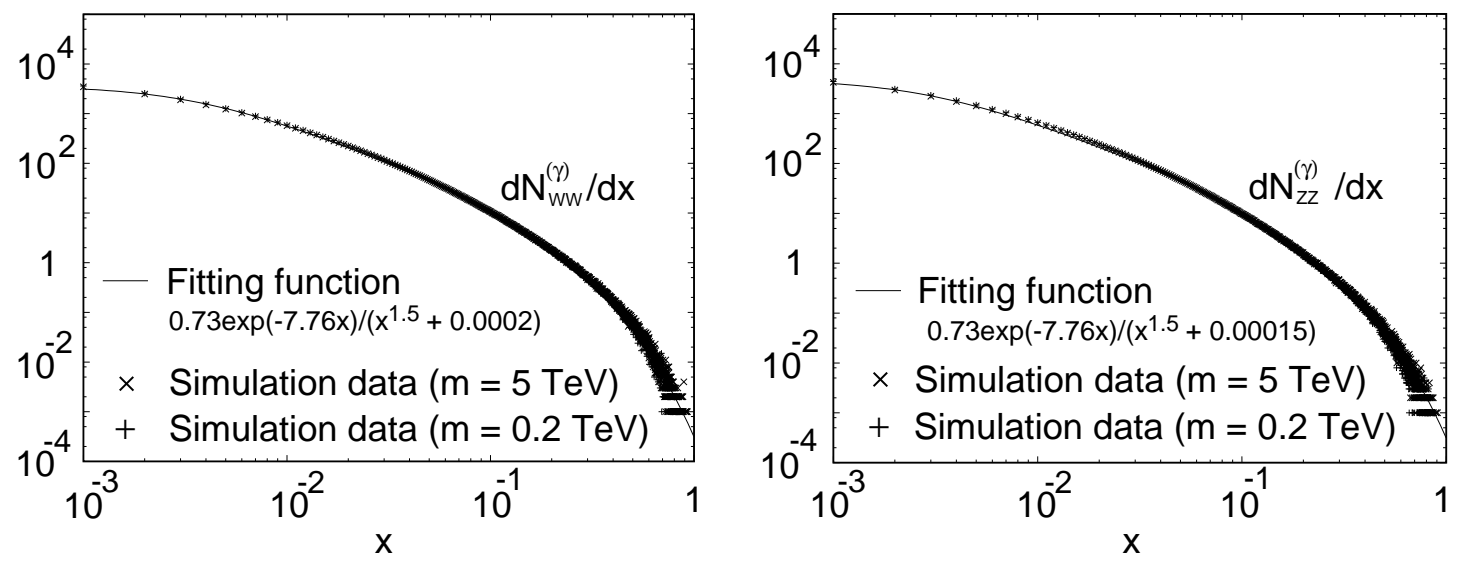

Figure 10: Fragmentation functions of $W^{+} W^{-}$(the left figure) and $Z^{0} Z^{0}$ (the right figure). The cross points are the simulation data by HERWIG. The fitting functions are also depicted in these figures as solid lines.

The angular resolution of the detector, $\Delta \Omega$, is taken to be $10^{-3}$ in this paper, which is a typical value for the ACT detectors. The information of the dark matter density profile is included in a dimensionless function,

$$
\bar{J}=\int_{\text {line of sight }} \frac{d l(\theta)}{8.5 \mathrm{kpc}} \int_{\Delta \Omega} \frac{d \Omega}{\Delta \Omega}\left(\frac{\rho}{0.3 \mathrm{GeVcm}^{-3}}\right)^{2} .
$$

We need three quantities for evaluating the gamma ray fluxes; the dark matter annihilation cross sections to final states $f\left(\langle\sigma v\rangle_{f}\right)$, the fragmentation functions $\left(d N_{f}^{\gamma} / d E\right)$, and the mass density profile of the dark matter $(\rho)$. The cross sections are obtained in the previous section. We discuss the fragmentation functions and the mass density profile in the following subsections.

\section{Fragmentation function}

Since we focus on the $\mathrm{SU}(2)_{L}$ non-singlet dark matter, such as the Wino- or Higgsino-like neutralino in the MSSM, the continuum gamma ray signal come mainly from the the dark matter annihilation modes into $W^{+} W^{-}$and $Z^{0} Z^{0}$. We thus need two fragmentation functions, $N_{W^{+} W^{-}}^{(\gamma)}$ and $N_{Z^{0} Z^{0}}^{(\gamma)}$. We simulate the photon spectrums from these weak gauge boson states by the HERWIG Monte Carlo code [31. We derive fitting functions from the simulated fragmentation functions for $m=200 \mathrm{GeV}$ and $m=5 \mathrm{TeV}$ by introducing the scaling variable $x=E / m$.

In Fig. 10, the HERWIG simulation data for the fragmentation functions are shown. In these figures, the fitting functions are also depicted as solid lines. The 


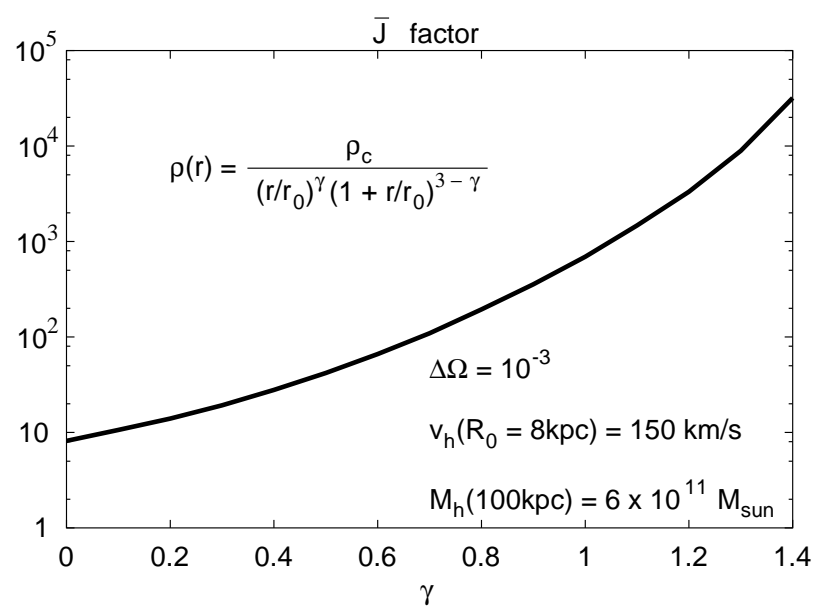

Figure 11: Dependence of $\bar{J}$ in Eq. (66) on $\gamma$ in the dark matter halo profile given in Eq. (69). We set the other parameters $\alpha$ and $\beta$ to be 1 and 3, respectively. The mass of the galaxy $M_{h}$ and the rotational velocity $v_{h}$, which are used to determine the parameters $\rho_{0}$ and $a$, are also shown in the figure.

functions are given by

$$
\frac{d N_{f}^{(\gamma)}}{d x}=\frac{0.73 e^{-7.76 x}}{x^{1.5}+c_{f}}
$$

where the parameter $c_{f}$ is $2 \times 10^{-4}$ for $f=W^{+} W^{-}$and $1.5 \times 10^{-4}$ for $f=Z^{0} Z^{0}$.

In the previous studies in Refs. [15], the cutoff parameter $c_{f}$ is not introduced in the fitting functions. However, the behaviors of the simulated fragmentation functions at $x \lesssim 10^{-(2-3)}$ are more moderate than the fitting functions with $c_{f}=0$. In this paper we consider cases of heavy EWIMPs $(\sim 10 \mathrm{TeV})$ and compare the predicted gamma ray fluxes with the EGRET data around $1 \sim 10 \mathrm{GeV}$. Thus, the effect of non-vanishing $c_{f}$ is not negligible.

The function $N_{\gamma \gamma}^{(\gamma)}$ for the line gamma ray flux is simply given by

$$
\frac{d N_{\gamma \gamma}^{(\gamma)}}{d E}=2 \delta(E-m),
$$

because the dark matter particle is almost at rest in the galactic halo.

3. Mass density profile of dark matter

The gamma ray flux depends strongly on the dark matter density profile, because it is proportional to the density squared. Many $N$-body simulations show that the 
dark matter halo profiles are given by a universal spherical functional form,

$$
\rho(r)=\frac{\rho_{0}}{(r / a)^{\gamma}\left[1+(r / a)^{\alpha}\right]^{(\beta-\gamma) / \alpha}},
$$

where $\alpha, \beta$ and $\gamma$ are the model parameters. After choosing the model parameters, $\rho_{0}$ and $a$ are uniquely determined by the mass and the rotational speed of the galaxy.

A famous and frequently used halo model is the Navarro, Frenk and White (NFW) profile [32, which corresponds to $(\alpha, \beta, \gamma)=(1,3,1)$ in Eq. (69). The profile is obtained by the numerical $N$-body simulation of point particles. Recently higher statistical simulations have been performed, and even higher values of $\gamma$ are obtained. For example, Moore et. al. found the profile corresponding to $(\alpha, \beta, \gamma)=(1,3,1.4 \sim$ 1.5) 33]. The halo profiles in all $N$-body simulations have cuspy structures and the density diverges at $r=0$. On the other hand, a no-cuspy model has been used for a long time, and the model parameters for the King-profile are $(\alpha, \beta, \gamma)=(1,3,0)$. It is argued that the rotation curve measurements of low surface brightness galaxies disfavor the cuspy profiles [34], though this disagreement may be resolved by taking into account the effect of halo triaxiality [35. This cusp/core problem is still under debate.

In Fig. 11, $\bar{J}$ in Eq. (66) is shown as a function of the model parameter $\gamma$. Here we fix $\alpha=1$ and $\beta=3$. We use the mass of galaxy interior to $100 \mathrm{kpc}$ $\left(M_{h}(100 \mathrm{kpc})=6 \times 10^{11} M_{\text {sun }}\right.$ with $M_{\text {sun }}$ the solar mass $)$ and the rotational speed at $r=8 \mathrm{kpc}\left(v_{h}(8 \mathrm{kpc})=150 \mathrm{~km} / \mathrm{s}\right)$ for the determination of $\rho_{0}$ and $a$ in Eq. (69). In

the figure $\bar{J}$ is sensitive to the value of $\gamma$. In the following, we use a moderate value $\bar{J}=500$, which is typical for the NFW profile.

\section{Line gamma ray flux from the galactic center}

We discuss the line gamma ray flux from the galactic center due to the EWIMP dark matter annihilation by using the cross sections derived in the previous section.

In Fig. 12, we show the line gamma fluxes from the galactic center in the cases of the triplet and doublet EWIMP dark matters. Here, we take the mass difference between the EWIMP and the charged partner as $\delta m=0.1,1,10 \mathrm{GeV}$. For the doublet EWIMP, $\delta m_{N}=2 \delta m$ is assumed. We also plot the flux obtained from the leading-order cross sections in perturbation by broken lines for comparison. In Fig. 13, we show the contour maps of the line gamma ray flux in the MSSM. The range of the MSSM parameters is the same as that for Figs. 6] and [ in the previous section. 

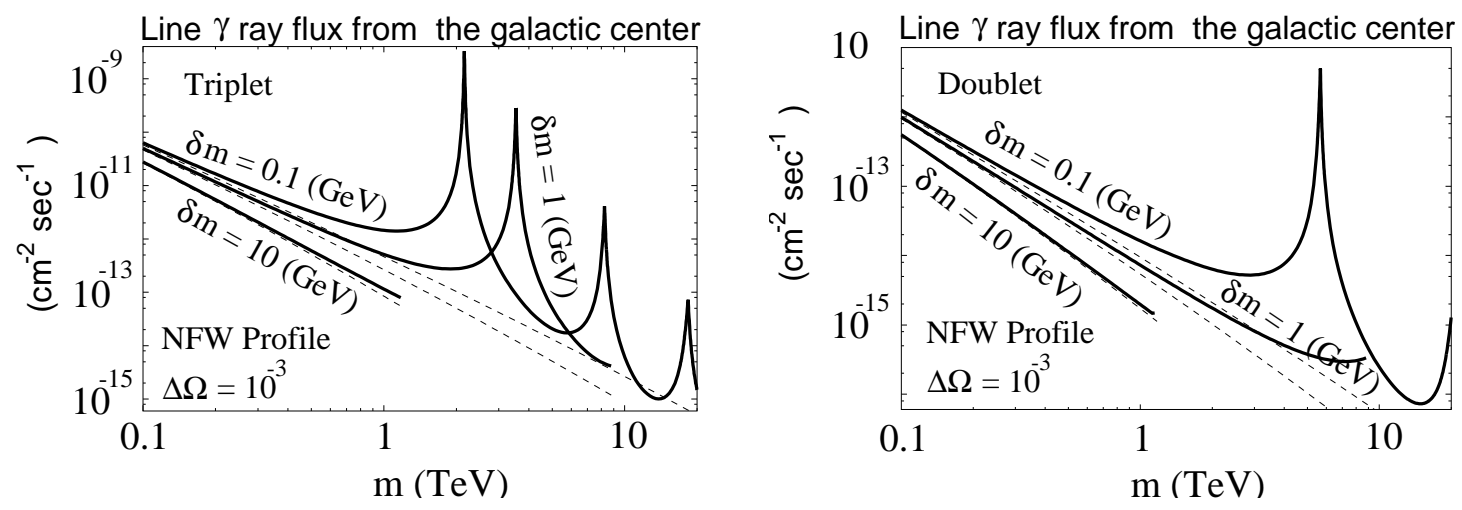

Figure 12: Line gamma ray flux from the galactic center in cases of the EWIMP dark matters. The left (right) figure is for the triplet (doublet) EWIMP. We take the average velocity of the dark matter $v / c=10^{-3}, \bar{J}=500$, which corresponds to the NFW profile, and $\Delta \Omega=10^{-3}$. The leading-order cross sections in perturbation are also shown as broken lines.

The large ACT detectors have high sensitivity for TeV-scale gamma rays. For example, MAGIC [13] and VERITAS [14] in the northern hemisphere might reach $10^{-14} \mathrm{~cm}^{-2} \mathrm{~s}^{-1}$ at the TeV scale while CANGAROO III [11] and HESS [12] in the southern hemisphere might reach $10^{-13} \mathrm{~cm}^{-2} \mathrm{~s}^{-1}$. From Figs. 12 and 13, it is found that these ACT detectors may cover broad regions in the parameter space.

It has been known that the line gamma ray signal is sensitive to the heavier dark matter with the mass of the order of $\mathrm{TeV}$, because the annihilation cross section at one-loop level is not suppressed by the dark matter mass if the dark matter has the $\mathrm{SU}(2)_{L}$ charge. Our studies reveal importance of the non-perturbative effects on the EWIMP annihilation cross section. After including the effects, the sensitivity of the line gamma ray signal to the heavier EWIMP dark matter is enhanced furthermore.

5. Continuum gamma ray flux from the galactic center

In addition to the line gamma rays, the EWIMP annihilation produces the continuum gamma rays, which come mainly from the decay of $\pi^{0}$ in the fragmentation of the final state particles. Though the number of photons in the continuum gamma rays is expected to be higher than that in the line gamma rays, its spectrum may lack the distinctive feature. Since the flux of the diffused gamma ray background, especially from the galactic center, is not well known, it is difficult to extract the annihilation signal $(S)$ from the background $(B)$ as far as $S<B$. The EGRET has 

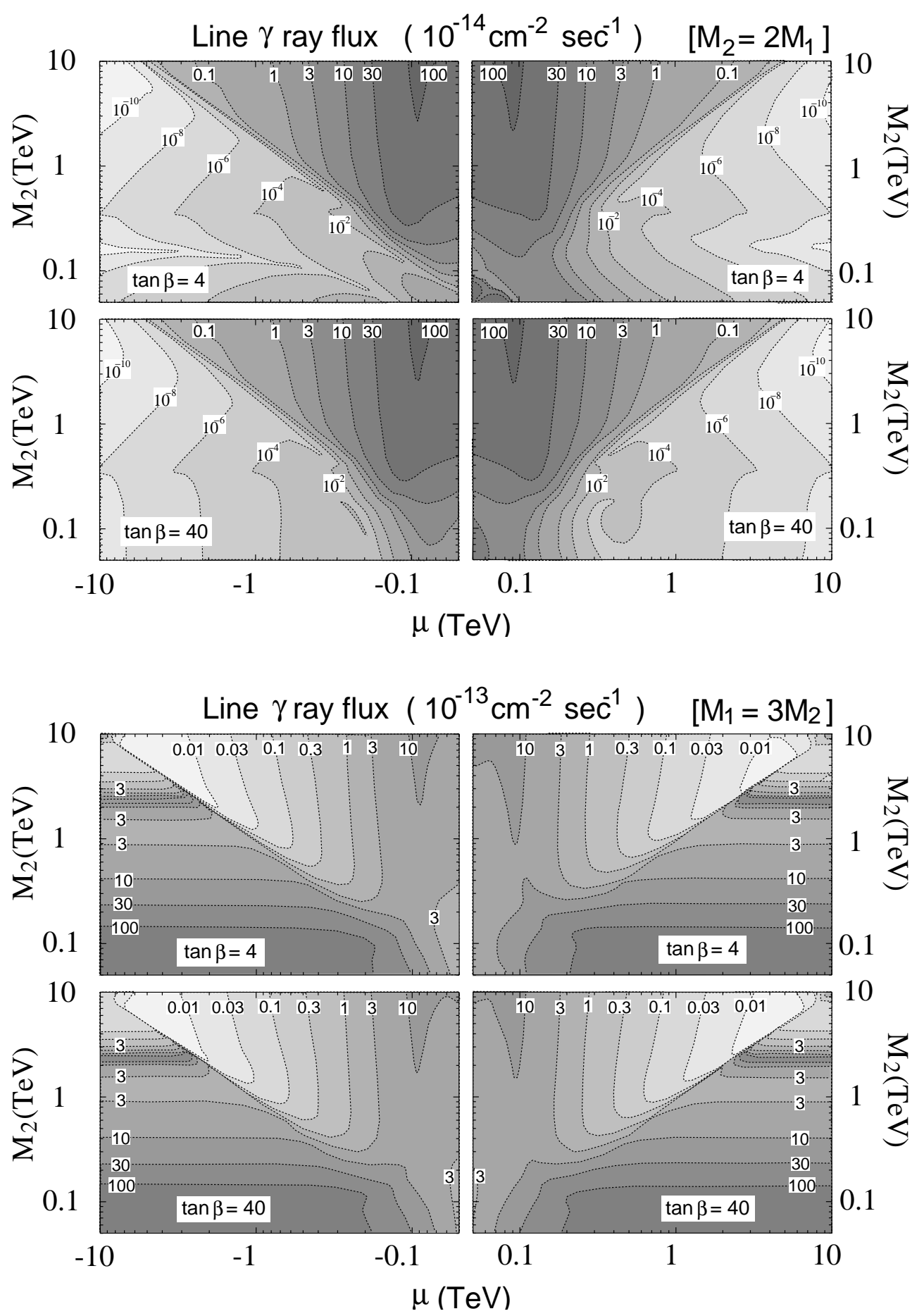

Figure 13: Contour maps of the line gamma ray flux in $\left(M_{2}, \mu\right)$ planes with $\tan \beta=4,40$ in the MSSM. $M_{1}=0.5 M_{2}$ is assumed in the four top figures while $M_{1}=3 M_{2}$ is assumed in the four bottom figures. These figures are depicted by using the NFW profile $(\bar{J}=500)$ and the angular resolution, $\Delta \Omega=10^{-3}$. 

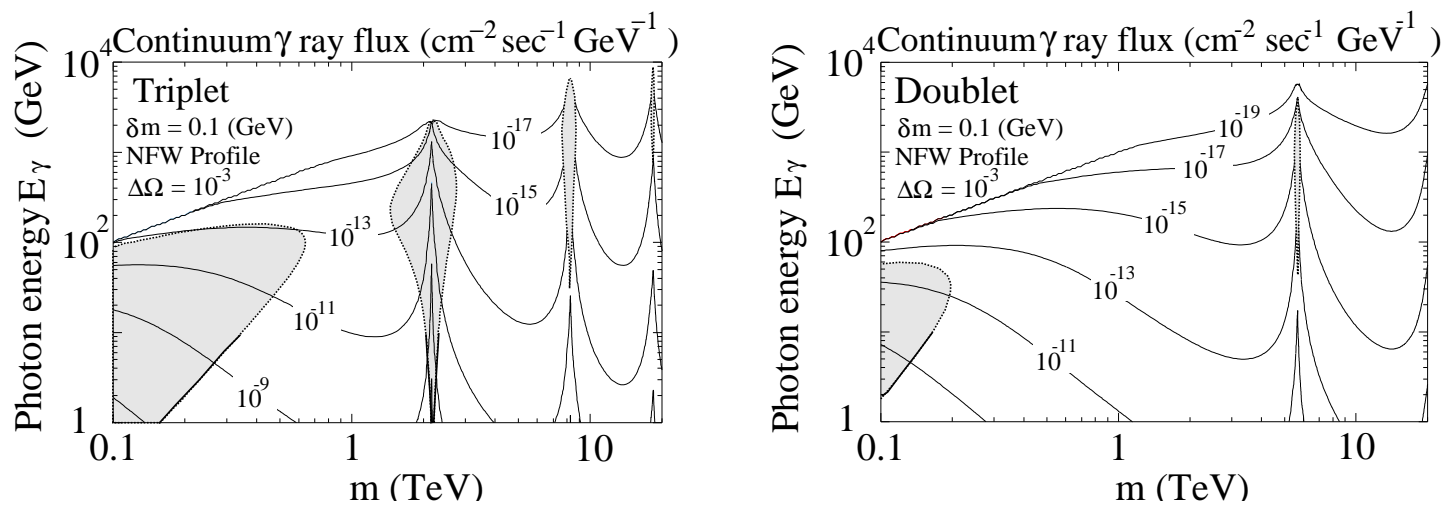

Figure 14: Contour plots of the continuum gamma ray flux from the galactic center in the unit of $\mathrm{cm}^{-2} \mathrm{sec}^{-1} \mathrm{GeV}^{-1}$. The left (right) plot corresponds to the triplet (doublet) EWIMP case. Here we take $\delta m=0.1 \mathrm{GeV}$, and other parameters are the same as those in Fig. 12. The shaded regions correspond to (Signal) $/($ Background) $>1$.

observed the diffused gamma ray emission from the galactic center up to about 10 $\mathrm{GeV}$ [36]. If the power law fall-off of the energy for the diffused gamma ray flux $\Psi_{B G}(E)$ is assumed, the background $B$ is evaluated from the EGRET result as [9],

$$
\frac{d \Psi_{B G}(E)}{d E}=9.1 \times 10^{-5}\left[\mathrm{~cm}^{-2} \sec ^{-1} \mathrm{GeV}^{-1}\right] \times\left(\frac{E}{1 \mathrm{GeV}}\right)^{-2.7} \Delta \Omega
$$

In Fig. 14 we show the contour plots of the continuum gamma ray fluxes from the galactic center for the triplet and doublet EWIMPs. Here we fix $\bar{J}=500, \Delta \Omega=10^{-3}$ and $\delta m=0.1 \mathrm{GeV}$. The shaded regions correspond to $S>B$, in which $B$ is given by Eq. (70). From this figure, it is found that even small regions around the resonances, in addition to areas with $m \sim 100 \mathrm{GeV}$, are already constrained from the EGRET measurement of the gamma ray flux with the energy $1 \sim 10 \mathrm{GeV}$.

In Fig. 15]regions excluded by the EGRET observation is shown in $\left(M_{2}, \mu\right)$ planes assuming the MSSM. The MSSM parameters are the same as in Fig. 13. In this figure we use $\bar{J}_{\mathrm{S}}$ with $\gamma=0,0.6,1$ and 1.4. The excluded regions become broader for larger $\gamma$.

In the near future the GLAST satellite 10] may observe gamma rays with energies in the range $1 \mathrm{GeV} \lesssim E \lesssim 300 \mathrm{GeV}$ if the flux is larger than about $10^{-10} \mathrm{~cm}^{-2} \mathrm{sec}^{-1}$. The ACT and GLAST detectors will constrain broader regions in the parameter space with $S>B$, or will find the signature of the dark matter.

\section{Summary}



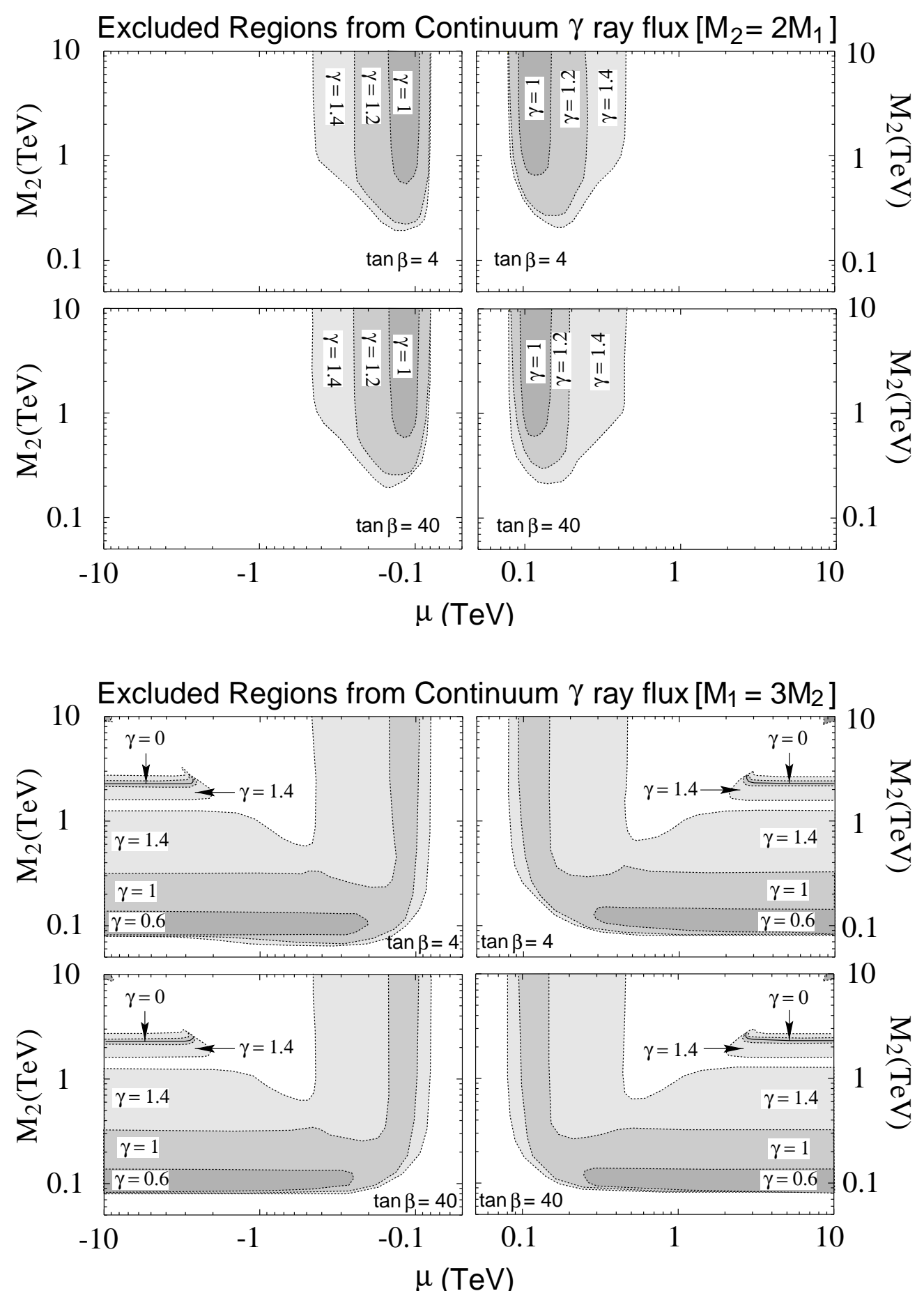

Figure 15: Contour maps of the excluded region by the EGRET measurement of the gamma ray flux from the galactic center in $\left(M_{2}, \mu\right)$ planes in the MSSM for different dark matter profiles. $M_{1}=0.5 M_{2}$ is assumed in the four top figures while it is $M_{1}=3 M_{2}$ in the four bottom figures. $\tan \beta=4,40$. These figures are depicted by using the $\bar{J}$ factors for $\gamma=0,0.6,1,1.4$. We also set the angular resolution to be $\Delta \Omega=10^{-3}$. 
In this paper we have calculated the pair annihilation cross sections of the EWIMP dark matters, which have an $\mathrm{SU}(2)_{L}$ charge of the standard model gauge group. The leading-order calculation of the cross sections in perturbation is no longer valid when the mass is heavy compared to the weak gauge bosons due to the threshold singularity coming from the mass degeneracy between the EWIMP and its $\mathrm{SU}(2)_{L}$ partner(s). The problem have been known for a while for the cases of the Wino and Higgsino-like dark matters in the MSSM. We have developed a method to take in the singularity and obtain the precise annihilation cross sections.

We find that if the mass of the EWIMP dark matter is larger than about $1 \mathrm{TeV}$, the attractive Yukawa potentials induced by the weak gauge boson exchanges have significant effects on the annihilation processes and the cross sections are enhanced by several orders of magnitude due to the zero-energy resonances under the potentials. As a result, the gamma ray flux from the galactic center due to the EWIMP annihilation is enhanced compared to the leading-order calculation in perturbation. The line gamma ray flux exceeds the typical sensitivity of the large ACT detectors such as CANGAROO III, HESS, VERITAS and MAGIC, $10^{-(13-14)} \mathrm{cm}^{-2} \mathrm{sec}^{-1}$, in the wide range of the MSSM parameters. We also calculated the continuum gamma ray flux from the EWIMP dark matter annihilation. The MSSM parameter space which is not consistent with the EGRET observation of the gamma rays with $1 \mathrm{GeV}$ $\lesssim E_{\gamma} \lesssim 10 \mathrm{GeV}$ is increased by the non-perturbative effects. The non-perturbative effect would also enhance anti-proton and positron fluxes from the dark matter annihilation. These will be discussed elsewhere [37.

Current observations of $\mathrm{TeV}$-scale gamma rays from the galactic center by the CANGAROO and HESS disagree each other in the spectrum. Once it is converged, the result may be used to constrain the EWIMP dark matter.

\section{Acknowledgments}

This work is supported in part by the Grant-in-Aid for Science Research, Ministry of Education, Science and Culture, Japan (No. 13135207 and 14046225 for JH and No. 14540260, 14046210 and 16081207 for MMN). MMN is also supported in part by a Grant-in-Aid for the 21st Century COE "Center for Diversity and Universality in Physics". 


\section{References}

[1] D. N. Spergel et al. [WMAP Collaboration], Astrophys. J. Suppl. 148, 175 (2003); C. L. Bennett et al., Astrophys. J. Suppl. 148, 1 (2003).

[2] For reviews, G. Jungman, M. Kamionkowski and K. Griest, Phys. Rept. 267, 195 (1996); L. Bergstrom, Rept. Prog. Phys. 63, 793 (2000); G. Bertone, D. Hooper and J. Silk, arXiv:hep-ph/0404175; C. Munoz, Int. J. Mod. Phys. A 19, 3093 (2004).

[3] J. R. Primack, Nucl. Phys. Proc. Suppl. 124, 3 (2003).

[4] J. Silk, K. A. Olive and M. Srednicki, Phys. Rev. Lett. 55, 257 (1985); T. K. Gaisser, G. Steigman and S. Tilav, Phys. Rev. D 34, 2206 (1986); S. Ritz and D. Seckel, Nucl. Phys. B 304, 877 (1988);

[5] G. F. Giudice and E. Roulet, Nucl. Phys. B 316, 429 (1989).

[6] H. U. Bengtsson, P. Salati and J. Silk, Nucl. Phys. B 346, 129 (1990); V. Berezinsky, A. Bottino and G. Mignola, Phys. Lett. B 325, 136 (1994); G. Jungman and M. Kamionkowski, Phys. Rev. D 51, 328 (1995); L. Bergstrom, J. Edsjo and P. Gondolo, Phys. Rev. D 55, 1765 (1997).

[7] E. A. Baltz, J. Edsjo, K. Freese and P. Gondolo, Phys. Rev. D 65, 063511 (2002); P. Ullio, JHEP 0106, 053 (2001); L. Bergstrom, J. Edsjo and P. Ullio, Phys. Rev. Lett. 87, 251301 (2001).

[8] L. Bergstrom and P. Gondolo, Astropart. Phys. 5, 263 (1996); J. R. Ellis, J. L. Feng, A. Ferstl, K. T. Matchev and K. A. Olive, Eur. Phys. J. C 24, 311 (2002).

[9] L. Bergstrom, P. Ullio and J. H. Buckley, Astropart. Phys. 9, 137 (1998).

[10] A. Morselli, SLAC-REPRINT-1997-111.

[11] K. Tsuchiya et al. [CANGAROO-II Collaboration], Astrophys. J. 606, L115 (2004).

[12] J. A. Hinton [The HESS Collaboration], New Astron. Rev. 48, 331 (2004).

[13] C. Baixeras [MAGIC Collaboration], Nucl. Phys. Proc. Suppl. 114 (2003) 247. 
[14] T. C. Weekes et al., arXiv:astro-ph/9706143.

[15] J. Hisano, S. Matsumoto and M. M. Nojiri, Phys. Rev. D 67, 075014 (2003); J. Hisano, S. Matsumoto and M. M. Nojiri, Phys. Rev. Lett. 92, 031303 (2004).

[16] D. S. Akerib et al. [CDMS Collaboration], Phys. Rev. D 68, 082002 (2003); A. Benoit et al., Phys. Lett. B 545, 43 (2002).

[17] H. E. Haber and G. L. Kane, Phys. Rept. 117 (1985) 75; H. P. Nilles, Phys. Rept. 110, 1 (1984).

[18] N. Arkani-Hamed and S. Dimopoulos, arXiv:hep-th/0405159, N. Arkani-Hamed, S. Dimopoulos, G. F. Giudice and A. Romanino, arXiv:hep-ph/0409232.

[19] L. Bergstrom and P. Ullio, Nucl. Phys. B 504, 27 (1997); Z. Bern, P. Gondolo and M. Perelstein, Phys. Lett. B 411, 86 (1997); P. Ullio and L. Bergstrom, Phys. Rev. D 57, 1962 (1998).

[20] W. E. Caswell and G. P. Lepage, Phys. Lett. B 167, 437 (1986).

[21] M. J. Strassler and M. E. Peskin, Phys. Rev. D 43, 1500 (1991).

[22] G. T. Bodwin, E. Braaten and G. P. Lepage, Phys. Rev. D 51, 1125 (1995) [Erratum-ibid. D 55, 5853 (1997)];

[23] H. C. Cheng, B. A. Dobrescu and K. T. Matchev, Nucl. Phys. B 543, 47 (1999).

[24] L. Randall and R. Sundrum, Nucl. Phys. B 557, 79 (1999); G. F. Giudice, M. A. Luty, H. Murayama and R. Rattazzi, JHEP 9812, 027 (1998).

[25] S. Profumo and C. E. Yaguna, Phys. Rev. D 70, 095004 (2004).

[26] E. W. Kolb and M. S. Turner, "The Early Universe,"; B. W. Lee and S. Weinberg, Phys. Rev. Lett. 39, 165 (1977); J. R. Ellis, J. S. Hagelin, D. V. Nanopoulos, K. A. Olive and M. Srednicki, Nucl. Phys. B 238, 453 (1984); P. Gondolo and G. Gelmini, Nucl. Phys. B 360, 145 (1991); M. Drees and M. M. Nojiri, Phys. Rev. D 47, 376 (1993).

[27] K. Enqvist and J. McDonald, Nucl. Phys. B 538, 321 (1999); T. Moroi and L. Randall, Nucl. Phys. B 570, 455 (2000). 
[28] K. Griest, Phys. Rev. D 38, 2357 (1988); M. Drees and M. Nojiri, Phys. Rev. D 48, 3483 (1993); B. Murakami and J. D. Wells, Phys. Rev. D 64, 015001 (2001); A. Djouadi, M. Drees, P. Fileviez Perez and M. Muhlleitner, Phys. Rev. D 65, 075016 (2002).

[29] J. Hisano, S. Matsumoto, M. M. Nojiri and O. Saito, arXiv:hep-ph/0407168.

[30] L.D. Landau, Quantum Mechanics: Non-Relativistic Theory, Volume 3, Third Edition (Elsevier Science Ltd).

[31] G. Corcella, I.G. Knowles, G. Marchesini, S. Moretti, K. Odagiri, P. Richardson, M.H. Seymour and B.R. Webber, JHEP 0101, 010 (2001).

[32] J. F. Navarro, C. S. Frenk and S. D. M. White, Astrophys. J. 462, 563 (1996); J. F. Navarro, C. S. Frenk and S. D. M. White, Astrophys. J. 490, 493 (1997).

[33] B. Moore, S. Ghigna, F. Governato, G. Lake, T. Quinn, J. Stadel and P. Tozzi, Astrophys. J. 524, L19 (1999); J. Diemand, B. Moore and J. Stadel, Mon. Not. Roy. Astron. Soc. 353, 624 (2004).

[34] See the references in W.J.G. de Blok, the proceedings of IAU Symposium 220: Dark Matter in Galaxies, Sydney, Australia, 21-25 Jul 2003, astro-ph/0311117.

[35] E. Hayashi et al., arXiv:astro-ph/0408132.

[36] S. D. Hunger et al., Astrophys. J. 481, 205 (1997).

[37] J. Hisano, S. Matsumoto, M. M. Nojiri and O. Saito, in preparation. 